\title{
The Solar Orbiter EUI instrument: The Extreme Ultraviolet Imager
}

P. Rochus ${ }^{1}$, F. Auchère ${ }^{2}$, D. Berghmans ${ }^{3}$, L. Harra ${ }^{4}$, W. Schmutz ${ }^{5}$, U. Schühle ${ }^{6}$, P. Addison ${ }^{4}$, T. Appourchaux ${ }^{2}$, R. Aznar Cuadrado ${ }^{6}$, D. Baker ${ }^{4}$, J. Barbay ${ }^{2}$, D. Bates ${ }^{4}$, A. BenMoussa ${ }^{3}$, M. Bergmann ${ }^{6}$, C. Beurthe ${ }^{7}$, B. Borgo ${ }^{2}$, K. Bonte ${ }^{3}$, M. Bouzit ${ }^{2}$, L. Bradley ${ }^{4}$, V. Büchel ${ }^{5}$, E. Buchlin ${ }^{2}$, J. Büchner ${ }^{6}$, F. Cabé2 ${ }^{2}$ L. Cadiergues ${ }^{9}$, M. Chaigneau ${ }^{2}$, B. Chares ${ }^{6}$, C. Choque Cortez ${ }^{2}$, P. Coker ${ }^{4}$, M. Condamin ${ }^{2}$, S. Coumar ${ }^{7}$, W. Curdt ${ }^{6}$, J. Cutler $^{4}$, D. Davies ${ }^{4}$, G. Davison ${ }^{4}$, J.-M. Defise ${ }^{1}$, G. Del Zanna ${ }^{8}$, F. Delmotte ${ }^{7}$, V. Delouille ${ }^{3}$, L. Dolla $^{3}$, C. Dumesnil ${ }^{2}$, F. Dürig ${ }^{5}$, R. Enge ${ }^{6}$, S. François ${ }^{2}$, J.-J. Fourmond ${ }^{2}$, J.-M. Gillis ${ }^{1}$, B. Giordanengo ${ }^{3}$, S. Gissot ${ }^{3}$, L. M. Green ${ }^{4}$, N. Guerreiro ${ }^{5}$, A. Guilbaud ${ }^{7}$, M. Gyo ${ }^{5}$, M. Haberreiter ${ }^{5}$, A. Hafiz ${ }^{4}$, M. Hailey ${ }^{4}$, J.-P. Halain ${ }^{1}$, J. Hansotte ${ }^{2}$, C. Hecquet ${ }^{7}$, K. Heerlein ${ }^{6}$, M.-L. Hellin ${ }^{1}$, S. Hemsley ${ }^{4}$, A. Hermans ${ }^{1}$, V. Hervier ${ }^{2}$, J.-F. Hochedez ${ }^{3}$, Y. Houbrechts ${ }^{1}$, K. Ihsan ${ }^{4}$, L. Jacques ${ }^{1}$, A. Jérôme ${ }^{7}$, J. Jones ${ }^{4}$, M. Kahle ${ }^{6}$, T. Kennedy ${ }^{4}$, M. Klaproth ${ }^{6}$, M. Kolleck ${ }^{6}$, S. Koller ${ }^{5}$, E. Kotsialos ${ }^{4}$, E. Kraaikamp ${ }^{3}$, P. Langer ${ }^{5}$, A. Lawrenson ${ }^{4}$, J.-C. Le Clech' ${ }^{2}$, C. Lenaerts ${ }^{1}$, S. Liebecq ${ }^{1}$, D. Linder ${ }^{4}$, D. M. Long ${ }^{4}$, B. Mampaey ${ }^{3}$, D. Markiewicz-Innes ${ }^{6}$, B. Marquet ${ }^{1}$, E. Marsch ${ }^{6}$, S. Matthews ${ }^{4}$, E. Mazy ${ }^{1}$, A. Mazzoli ${ }^{1}$, S. Meining ${ }^{6}$, E. Meltchakov ${ }^{7}$, R. Mercier ${ }^{7}$, S. Meyer ${ }^{6}$, M. Monecke ${ }^{6}$, F. Monfort ${ }^{1}$, G. Morinaud ${ }^{2}$, F. Moron ${ }^{7}$, L. Mountney ${ }^{4}$, R. Müller ${ }^{6}$, B. Nicula ${ }^{3}$, S. Parenti ${ }^{2,3}$, H. Peter ${ }^{6}$, D. Pfiffner ${ }^{5}$, A. Philippon ${ }^{2}$, I. Phillips ${ }^{4}$, J.-Y. Plesseria ${ }^{1}$, E. Pylyser ${ }^{3}$, F. Rabecki ${ }^{1}$, M.-F. Ravet-Krill ${ }^{7}$, J. Rebellato ${ }^{7}$, E. Renotte ${ }^{1}$, L. Rodriguez ${ }^{3}$, S. Roose ${ }^{1}$, J. Rosin ${ }^{1}$, L. Rossi ${ }^{1}$, P. Roth ${ }^{7}$, F. Rouesnel ${ }^{2}$, M. Roulliay ${ }^{7}$, A. Rousseau ${ }^{4}$, K. Ruane ${ }^{4}$, J. Scanlan ${ }^{4}$, P. Schlatter ${ }^{5}$, D. B. Seaton ${ }^{3, \star}$, K. Silliman ${ }^{4}$, S. Smit ${ }^{4}$, P. J. Smith ${ }^{4}$, S. K. Solanki ${ }^{6}$, M. Spescha ${ }^{5}$, A. Spencer ${ }^{4}$, K. Stegen ${ }^{3}$, Y. Stockman ${ }^{1}$, N. Szwec ${ }^{2}$, C. Tamiatto ${ }^{2}$, J. Tandy ${ }^{4}$, L. Teriaca ${ }^{6}$, C. Theobald ${ }^{4}$, I. Tychon ${ }^{1}$, L. van Driel-Gesztelyi ${ }^{4}$, C. Verbeeck ${ }^{3}$, J.-C. Vial ${ }^{2}$, S. Werner ${ }^{6}$, M. J. West ${ }^{3}$, D. Westwood ${ }^{4}$, T. Wiegelmann ${ }^{6}$, G. Willis ${ }^{4}$, B. Winter ${ }^{4}$, A. Zerr ${ }^{6}$, X. Zhang ${ }^{2}$, and A. N. Zhukov ${ }^{3}$

1 Centre Spatial de Liège, Université de Liège, Av. du Pré-Aily B29, 4031 Angleur, Belgium

2 Institut d'Astrophysique Spatiale, CNRS, Univ. Paris-Sud, Université Paris-Saclay, Bât. 121, 91405 Orsay, France

3 Royal Observatory of Belgium, Ringlaan -3- Av. Circulaire, 1180 Brussels, Belgium e-mail: david.berghmans@oma.be

${ }^{4}$ UCL-Mullard Space Science Laboratory, Holmbury St. Mary, Dorking, Surrey RH5 6NT, UK

5 Physikalisch-Meteorologisches Observatorium Davos, World Radiation Center, 7260 Davos Dorf, Switzerland

6 Max Planck Institute for Solar System Research, Justus-von-Liebig-Weg 3, 37077 Göttingen, Germany

7 Laboratoire Charles Fabry, Institut d'Optique Graduate School, Université Paris-Saclay, 91127 Palaiseau Cedex, France

8 Centre for Mathematical Sciences, University of Cambridge, Wilberforce Road, Cambridge CB3 OWA, UK

9 Centre National d'Etudes Spatiales, 18 avenue Edouard Belin, 31401 Toulouse Cedex 9, France

Received 10 September 2019 / Accepted 5 December 2019

\section{ABSTRACT}

Context. The Extreme Ultraviolet Imager (EUI) is part of the remote sensing instrument package of the ESA/NASA Solar Orbiter mission that will explore the inner heliosphere and observe the Sun from vantage points close to the Sun and out of the ecliptic. Solar Orbiter will advance the "connection science" between solar activity and the heliosphere.

Aims. With EUI we aim to improve our understanding of the structure and dynamics of the solar atmosphere, globally as well as at high resolution, and from high solar latitude perspectives.

Methods. The EUI consists of three telescopes, the Full Sun Imager and two High Resolution Imagers, which are optimised to image in Lyman- $\alpha$ and EUV $(17.4 \mathrm{~nm}, 30.4 \mathrm{~nm})$ to provide a coverage from chromosphere up to corona. The EUI is designed to cope with the strong constraints imposed by the Solar Orbiter mission characteristics. Limited telemetry availability is compensated by state-of-theart image compression, onboard image processing, and event selection. The imposed power limitations and potentially harsh radiation environment necessitate the use of novel CMOS sensors. As the unobstructed field of view of the telescopes needs to protrude through the spacecraft's heat shield, the apertures have been kept as small as possible, without compromising optical performance. This led to a systematic effort to optimise the throughput of every optical element and the reduction of noise levels in the sensor.

Results. In this paper we review the design of the two elements of the EUI instrument: the Optical Bench System and the Common Electronic Box. Particular attention is also given to the onboard software, the intended operations, the ground software, and the foreseen data products.

Conclusions. The EUI will bring unique science opportunities thanks to its specific design, its viewpoint, and to the planned synergies with the other Solar Orbiter instruments. In particular, we highlight science opportunities brought by the out-of-ecliptic vantage point of the solar poles, the high-resolution imaging of the high chromosphere and corona, and the connection to the outer corona as observed by coronagraphs.

Key words. Sun: UV radiation - Sun: transition region - Sun: corona - space vehicles: instruments - telescopes instrumentation: high angular resolution

^ Now at: CIRES, Univ. of Colorado, Boulder, Colorado, USA. 


\section{Introduction: Instrument overview}

The ESA Solar Orbiter mission is designed to determine how the Sun creates and controls the heliosphere (Garcia-Marirrodriga et al. 2020). The spacecraft will bring a combination of in situ (Horbury et al. 2020) and remote-sensing instruments (Auchère et al. 2020) out of the ecliptic $\left(>30^{\circ}\right)$ and close to the Sun $(<0.3 \mathrm{AU})$, to answer the following four questions (Müller et al. 2020):

1. What drives the solar wind and where does the coronal magnetic field originate?

2. How do solar transients drive heliospheric variability?

3. How do solar eruptions produce energetic particle radiation that fills the heliosphere?

4. How does the solar dynamo work and drive connections between the Sun and the heliosphere?

As part of the remote-sensing instrument package of Solar Orbiter, the Extreme Ultraviolet Imager (EUI) will contribute to answering all four questions through the observation of structures and events in the solar atmosphere. The EUI instrument is composed of two units, the Optical Bench System (OBS) and the Common Electronics Box (CEB), which are linked by an interconnecting harness.

The OBS (Fig. 1) is a common structure holding the three telescopes with mirrors, cameras, filter wheels, filters, and door mechanism subsystems. These three complementary telescopes will collectively cover the range from the chromosphere to the outer corona, and image this range globally, at very high resolution and from high-latitude perspectives. The two High Resolution Imagers (HRIs) are two-mirror optical systems while the Full Sun Imager (FSI) is a one-mirror telescope, each working in near normal incidence. Spectral selection is obtained with specific multi-layered coatings on the mirrors, complemented by filters that reject the visible and infrared radiation.

The (E)UV photons are collected on $\mathrm{CMOS}^{1}$ Active Pixel Sensors (APS) of $3072 \times 3072$ pixels of $10 \mu \mathrm{m}$ each. For the HRI channels the sensors are windowed to $2048 \times 2048$ illuminated pixels. The FSI and the HRI $\mathrm{EUV}_{\mathrm{Ev}}$ use the APS sensor in a backside thinned configuration, while the $\mathrm{HRI}_{\text {Lya }}$ uses the APS sensor in a front-sided configuration in conjunction with an image intensifier. For each detector pixel, the resulting signal is proportional to the solar flux corresponding to the small viewing angle of the pixel in the given pass band. The electrical signals are converted to digital numbers in the front-end camera electronics, before being compressed and stored in the CEB. The CEB (Fig. 2) operates the instrument and provides the data handling (processing and compression) of the three telescopes.

The EUI OBS and EUI CEB are both mounted on the so-called payload "MY" panel (a large optical bench) on the $-Y$ side of the spacecraft, together with other remote-sensing instruments (Garcia-Marirrodriga et al. 2020). The EUI baffles pierce through cut-outs in the top floor "PX" panel of the spacecraft ( $+X$ side) where they connect to the corresponding feedthroughs of the spacecraft heat shield (see Fig. 3). The two HRI apertures are protected by a common heat shield door. The FSI aperture is protected by a second, separate heat shield door. In addition, each telescope has its own instrument door protecting the optical entrance aperture. Operating an EUI telescope thus requires that both the corresponding heat shield door and instrument door are opened.

\footnotetext{
1 Complementary Metal-Oxide-Semiconductor.
}

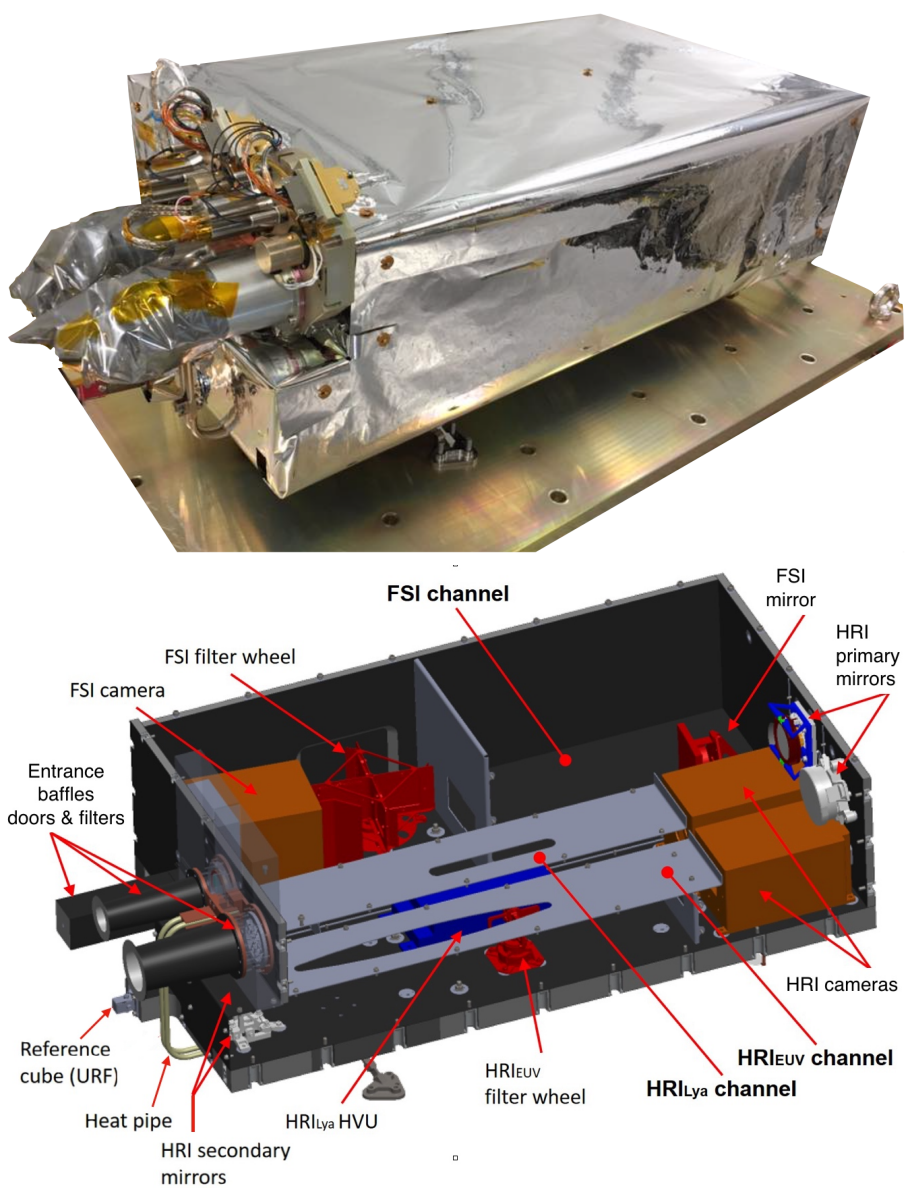

Fig. 1. Photo (top) and schematic (bottom) of the EUI OBS showing internal subsystems.

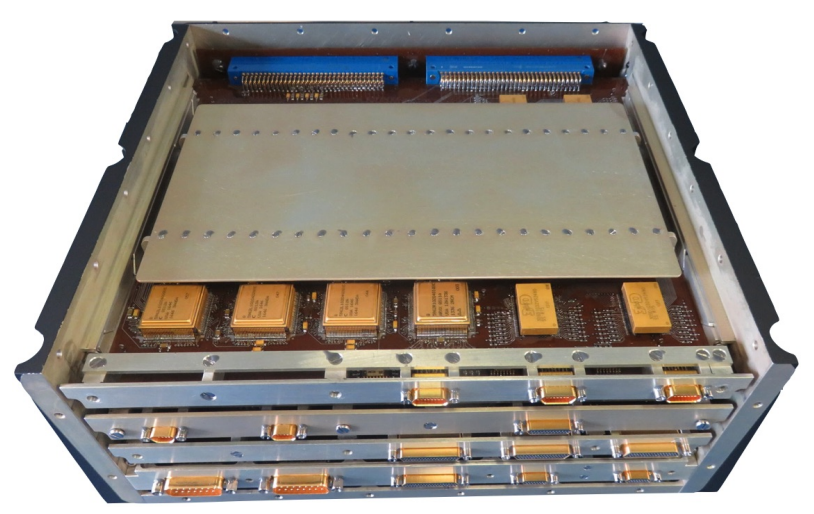

Fig. 2. CEB of EUI with the top and front panels removed.

\section{Scientific objectives}

In the following three sections we briefly highlight some science questions that EUI will be able to address thanks to the combination of its specific design, the unique orbit that Solar Orbiter will embark on, and to joint campaigns with the other Solar Orbiter instruments.

\subsection{The transition from corona to the heliosphere}

The large-scale structure of the low corona is determined by active regions, filament channels and coronal holes whose presence and location have a clear evolution over the solar cycle. 


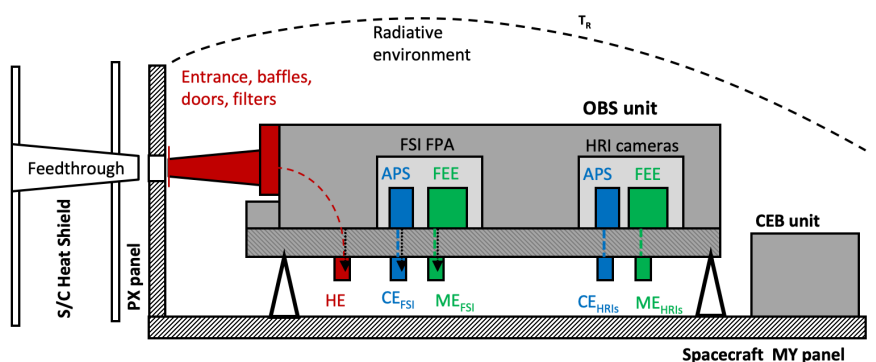

Fig. 3. EUI thermal interfaces overview showing the HE, ME, and CE connections.

Higher up in the corona, streamers and pseudo streamers, which arch over the active regions and filaments, stretch out into the heliospheric plasma sheet. This magnetic connection region between the corona and the heliosphere is of particular physical interest because it is here that the structures change from being magnetically dominated to being controlled by the plasma outflow.

When trying to determine the coronal foot-point of features observed by the in situ instruments, the transition from corona to heliosphere introduces significant uncertainty as the extrapolations from the photosphere have to be mapped to a Parkerspiral-type configuration. Deciphering this connection is thus of primordial importance for Solar Orbiter connection science. This "transition" corona (approximately one to three solar radii above the solar limb) is poorly studied as it corresponds to the gap in the field of view (FOV) between most previous EUV imagers and coronagraphs (Slemzin et al. 2008; Masson et al. 2014; Reva et al. 2016; O'Hara et al. 2019). The EUI Full Sun Imager (FSI) will be imaging alternatively in the $17.4 \mathrm{~nm}$ and $30.4 \mathrm{~nm}$ EUV pass-bands, with a $3.8^{\circ} \times 3.8^{\circ} \mathrm{FOV}$, corresponding to $\left(14.3 R_{\odot}\right)^{2}$ at $1 \mathrm{AU}$ and still $\left(4.0 R_{\odot}\right)^{2}$ at perihelion. This FOV is unprecedentedly large for a coronal EUV imager and will have a significant overlap (Auchère et al. 2020) with the Solar Orbiter coronagraph Metis (Antonucci et al. 2020).

Recent image mosaics collected by the Solar Ultraviolet Imager (SUVI) during exceptional off-points of the Geostationary Operational Environmental Satellite (GOES; Tadikonda et al. 2019, see Fig. 4) reveal that coronal structures indeed extend to several solar radii. Unexpected structures have been observed in the transition corona by the $\mathrm{HeCOR}^{2}$ and $\mathrm{SCORE}^{3}$ coronagraphs (FSI and METIS prototypes, Auchère et al. 2007; Romoli et al. 2007) on the HERSCHEL ${ }^{4}$ sounding rocket (see online $^{5}$ and Moses et al. 2020) and by the SWAP 6 EUV imager (Seaton et al. 2013b). In contrast to SWAP, the FSI detector will be cooled to $-40^{\circ} \mathrm{C}$, thus allowing deep exposures that are impossible to achieve with SWAP.

The large FSI FOV is designed to guarantee that even for maximal off-points by Solar Orbiter, the full solar disc remains in the FOV. The FSI will thus provide a view on the global morphology of the solar atmosphere (active regions, coronal holes, quiet Sun), thereby setting the scene in which the first Solar Orbiter science question above will be settled. As required for the second and third science question above, FSI will help us to understand the initial phase of solar eruptive processes (Green

\footnotetext{
2 Helium CORonagraph.

Solar CORonograph Experiment.

4 HElium Resonant Scattering in the Corona and HELiosphere.

http://metis.oato.inaf.it/score.html

6 Sun Watcher using Active Pixel System detector and Image Processing.
}

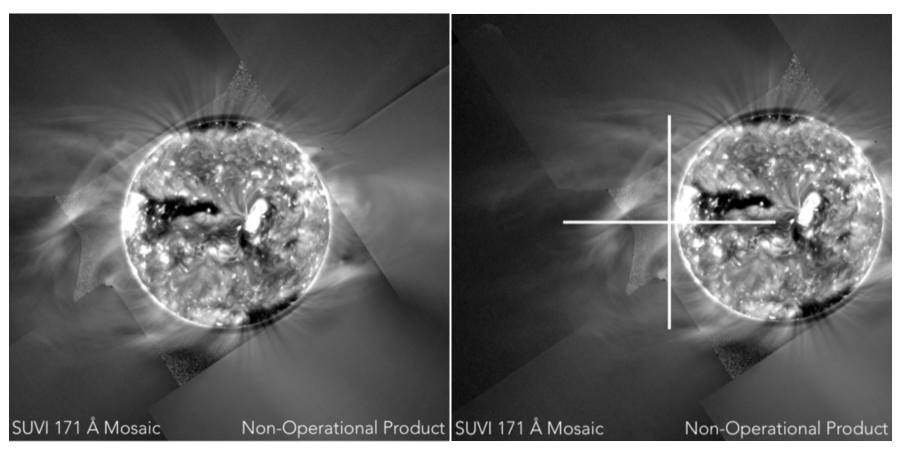

Fig. 4. Mosaics created by a series of off-pointings by the GOES/SUVI instrument, demonstrating EUV structures up to several solar radii (adapted from Tadikonda et al. 2019). The FOV shown in this figure corresponds to the FSI FOV at perihelion. Even with extreme off-pointing to the solar limb (right panel, white cross), the full solar disc remains in the FOV.

et al. 2018) such as flares, coronal mass ejections (CMEs) in the corona. For all these reasons, EUI will be an essential building block for the "connection science" to which all Solar Orbiter instruments will contribute.

\subsection{The solar atmosphere at high resolution}

After decades of investigations, there is still no full picture of why the corona, as the lower boundary of the heliosphere, actually exists: both the replenishment of energy as well as the replenishment of mass are not fully understood. How does the solar corona maintain its temperature and how is the solar wind mass loss compensated? Although the biggest energy releases (solar flares) and biggest mass expulsions (CMEs) can make enormous contributions, they are too sporadic in nature to explain the persistent existence of the corona. A general consensus exists that the crucial processes responsible for the formation of the corona must therefore be operating at small scales throughout the corona at all times.

The FSI is therefore accompanied by two high-resolution imagers (HRIs) to image fine structures and small-scale events in the solar atmosphere. The pass-bands of the HRIs are comparable to each of the pass-bands of FSI. The HRI imaging in the

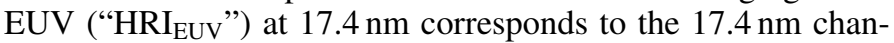
nel of FSI. The second HRI, which images in the Lyman- $\alpha$ line ("HRI Lya"), shares the same resonance formation process for hydrogen as the $30.4 \mathrm{~nm}$ channel of FSI for helium. In this way, EUI covers emission from the high chromosphere to the low corona, both at high resolution and at global scales. The HRIs have been designed to have a two-pixel resolution of 1 arcsec, which corresponds to a pixel footprint on the Sun of $(100 \mathrm{~km})^{2}$ during the perihelion observations. At this resolution, proper temporal sampling of features propagating at the sound speed of $150 \mathrm{~km} \mathrm{~s}^{-1}$ in a $1 \mathrm{MK}$ plasma implies an imaging cadence of the order of seconds. Both HRI cameras are capable of operating at 1 second imaging rates and faster in subfields. With a $2048 \times 2048$ pixel array, the HRIs will image a $17^{\prime} \times 17^{\prime} \mathrm{FOV}$, corresponding to $\left(1 R_{\odot}\right)^{2}$ when imaging at $1 \mathrm{AU}$ and $\left(0.28 R_{\odot}\right)^{2}$ at perihelion.

Until now, such temporal and spatial resolution has only been achieved by imagers on short duration sounding rockets such as Hi-C (Kobayashi et al. 2014) for coronal imaging and VAULT (Korendyke et al. 2001; Vourlidas et al. 2010) for Lyman- $\alpha$ imaging. Such observations have yielded exciting new results but remain hampered by small event number statistics: Hi-C was 
capable of resolving for the first time braiding of loop strands, but only two cases could be identified (Cirtain et al. 2013). Pant et al. (2015) studied flows and waves in a braided structure but they were limited to the $200 \mathrm{~s}$ duration of the Hi-C dataset. VAULT observations of an individual spicule (Chintzoglou et al. 2018) combined with IRIS (De Pontieu et al. 2014) data showed the spicule as a moving heating front without the need to assume any mass flow.

To obtain the richest possible datasets, the EUI HRI telescopes will be operated in joint campaigns with the Solar Orbiter spectrograph SPICE (SPICE Consortium 2020) and the PHI high-resolution telescope (HRT, Solanki et al. 2020). Telemetry limitations will not allow continuous HRI operations but nevertheless HRI image sequences of different targets will be taken regularly in different phases of the solar cycle and from increasing solar latitude perspectives. The HRI image sequences will provide unprecedented opportunities to study the fine structure in the solar low corona (loops, filaments, etc.) and the dynamics (waves, flows, jets, nano-flares, etc.) therein. A specific science objective of the EUI HRI telescopes is to understand the physical mechanisms producing and operating in the hot solar atmosphere, as this is the driving boundary of the whole heliosphere.

\subsection{The high-latitude perspective}

The EUI instrument will bring unique science opportunities not only because of its own design but also because of the unique orbit the platform embarks on (Garcia-Marirrodriga et al. 2020). With the help of a number of Venus gravity assist manoeuvres, the orbit of Solar Orbiter will gradually be lifted out of the plane of the ecliptic. More than $30^{\circ}$ solar latitude will be reached, which is sufficient to observe a solar pole entirely in a single image.

It is well known that the solar activity cycle is driven by a global dynamo, but it has been difficult to constrain this due to the lack of polar observations. In addition, there also exists evidence for a local dynamo, which can re-process magnetic field (Stenflo 2013). The particular magnetic field distribution in polar coronal holes implies that there could be differences in the behaviour and activity in the polar atmosphere as compared to lower-latitude regions. Harra et al. (2015) carried out a longterm study of polar regions with Hinode EIS and SDO AIA and found that the coronal non-thermal velocities measured in the polar region remained unchanged during the cycle, which was interpreted as a local dynamo effect. The number of bright points also remained unchanged.

The EUI will be able to explore the polar view of the convective cell boundaries using $\mathrm{HRI}_{\text {Lya }}$, and investigate bright points, jets, plumes, and spicules in the polar region using both HRIs during Solar Orbiter off-points. Polar features observed from high latitude can be compared stereoscopically with EUV imagers in the ecliptic plane. "Earth-view" observations indicate that statistics of small-scale dynamics remain constant throughout the cycle. The Solar Orbiter out-of-ecliptic view will undoubtedly transform our view of the poles and activity there. This will be done together with PHI, which will measure the polar magnetic field from an unprecedented point of view, and SPICE, which will measure the thermal dynamics, and Doppler and non-thermal velocities in these same structures in order to provide a comprehensive view on how the magnetic field dynamics transfer the magnetic energy to the solar plasma and produce these dynamical structures.

The fast solar wind emanates from the poles, and there will be many opportunities for comparison between EUI images and in situ observations of the fast solar wind, either at the Solar Orbiter location or elsewhere. In particular, dynamic features can be pursued, thereby uncovering the source(s) of the fast solar wind. Together with the Solar Orbiter PHI instrument, FSI will also play an important supportive role when the spacecraft is located in opposition to the Earth: imagery of the solar far-side showing the location of coronal holes and active regions will support 3D modelling of the solar coronal magnetic field contributing to the interpretation of the in situ solar wind data.

The other huge advantage of the out-of-ecliptic view will be the observations of the active latitudes from above. The FSI will be able to view eruptions from this novel angle. In addition, an occulter is available in FSI to allow exploration of the fainter corona further away from the solar surface.

\subsection{From constraints to technological innovation}

The specifics of Solar Orbiter as a deep-space and near-Sun mission imposes challenging constraints on the design and the operation of the EUI telescopes. In the following sections, we present how the EUI instrument was designed and built for maximal scientific exploitation in the presence of the imposed constraints.

For example, Solar Orbiter's proximity to the Sun during part of the orbit dictated that the telescope apertures in the heat shield must be small. This led to a systematic effort to optimise the throughput of every optical element and the reduction of noise levels in the sensor. Given the limited apertures, the selection of the different pass-bands of the telescopes was constrained to the brightest parts of the spectrum. These aspects are covered in Sect. 3 on the optical design, while the mechanical and thermal design is presented in Sect. 4.

The imposed power limitations and potentially harsh radiation environment led to the usage of novel CMOS-APS (see next section) sensors, previously pioneered in orbit for solar physics by the PROBA2/SWAP telescope (Seaton et al. 2013a; Halain et al. 2013). The telemetry available to EUI is orders of magnitude smaller than the data-production potential of the instrument. Maximisation of the science return of the instrument therefore implies maximising the science content of every bit of telemetry. The strategy to achieve this includes onboard processing, event selection, and state-of-the-art data compression. The hardware implementing all these functions is described in Sect. 5 on electronics.

The expected performance of the instrument is described in Sect. 8, and Sect. 9 lists the concepts of operations. Final conclusions are collected in Sect. 10.

\section{Optics}

\subsection{Full Sun Imager}

The FSI was designed according to the following top-level requirements:

- A FOV width of two solar diameters at perihelion so that the whole Sun can be seen when the spacecraft is pointed at the solar limb.

- An angular resolution of 10 arcsec.

- Two pass-bands centred on $30.4 \mathrm{~nm}$ and $17.4 \mathrm{~nm}$ to image the transition region and the EUV corona, with a spectral purity better than 80 and $90 \%$ respectively.

- Typical exposure times of a few seconds to be able to freeze the expected dynamics given the angular resolution.

These requirements, in combination with the efficiency of the available optics, dictated a relatively large entrance pupil. 
P. Rochus et al.: The Solar Orbiter EUI instrument: The Extreme Ultraviolet Imager
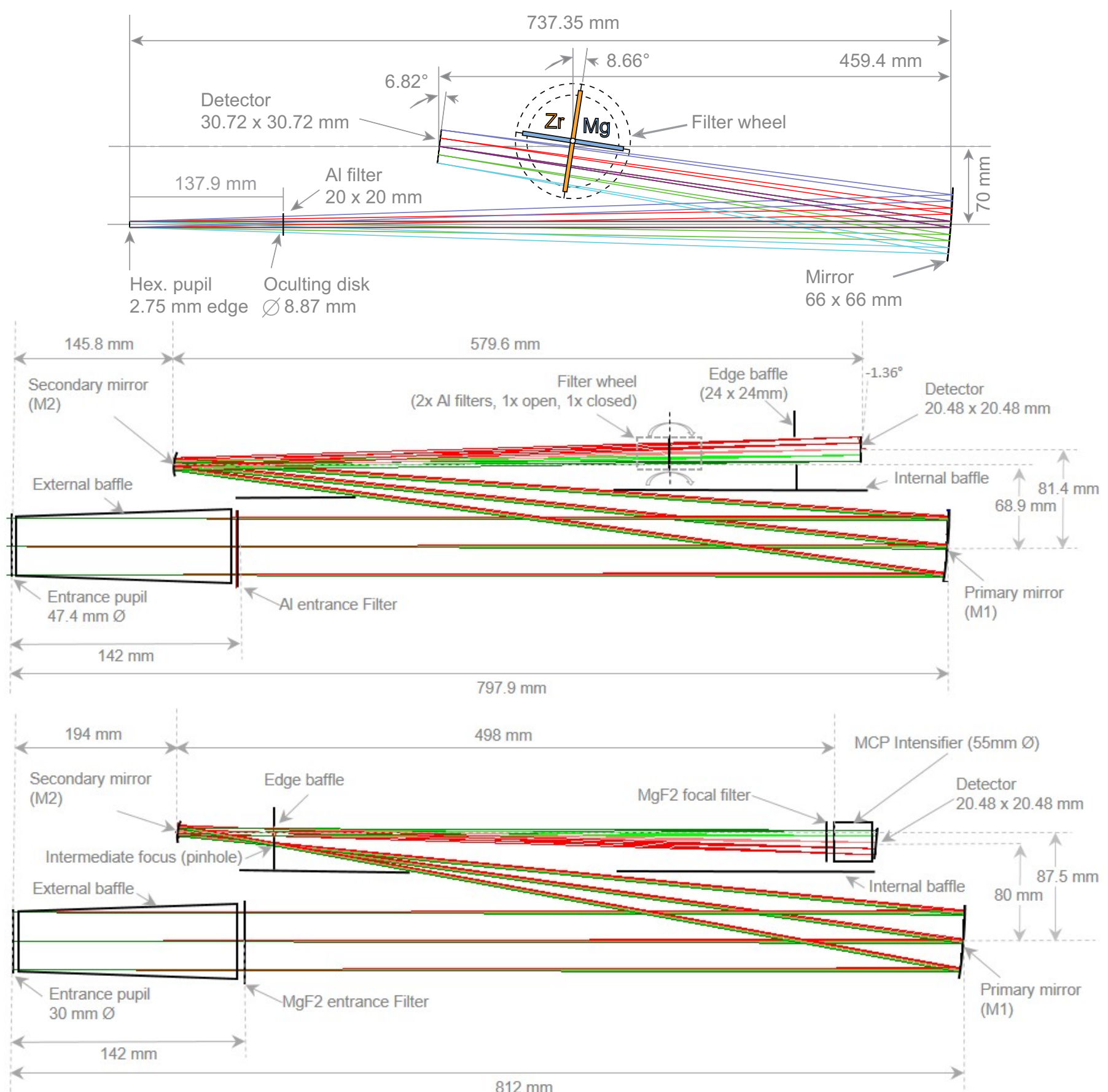

Fig. 5. Optical scheme with the positions and coordinates of the components of the three telescopes: the FSI (top), HRI $\mathrm{EUV}_{\mathrm{EUV}}($ middle), and HRI $\mathrm{Lya}$ (bottom).

It quickly became apparent that the desired angular resolution could not be achieved over the required $3.8^{\circ} \times 3.8^{\circ} \mathrm{FOV}$ using a traditional two-mirror telescope design within the allocated volume. The solution (Auchère et al. 2005) came from the adoption of a single-mirror design using a specially developed highefficiency coating, which allowed the pupil area to be reduced by a factor of four, enabling the required image quality to be maintained over the entire FOV.

The FSI optical design (see Fig. 5) therefore uses a single off-axis mirror (Herschelian telescope) making an image through a hexagonal (explained below) entrance pupil of $2.75 \mathrm{~mm}$ edge, located $737.35 \mathrm{~mm}$ from the vertex of the mirror. Similar designs have previously been flown on the CORONAS-F mission (e.g.
Kuzin et al. 2011). The optical prescription is given in Table 1. The mirror focal length is $462.5 \mathrm{~mm}$, which gives an average ${ }^{7}$ plate scale of $4.46^{\prime \prime}$ per $10 \mu \mathrm{m}$ pixel of the $3072 \times 3072$ APS detector. A $20 \mathrm{~mm} \times 20 \mathrm{~mm}$ aluminium filter of $150 \mathrm{~nm}$ thickness from the Luxel Corporation is positioned $140 \mathrm{~mm}$ behind the entrance pupil. This thin film, supported by a $0.4 \mathrm{~mm}$ pitch hexagonal mesh grid, rejects the visible and the infrared, letting only the EUV pass through (from the aluminium $L_{\mathrm{II}, \mathrm{III}}$ edge at $17 \mathrm{~nm}$ to about $80 \mathrm{~nm}$ ). This filter is of primary importance for the instrument to avoid contamination from visible light that is

7 There is a small difference of $2 \times 10^{-3}$ between the $X$ and $Y$ axes, which corresponds to a few pixels at the edge of the FOV. 
Table 1. Nominal characteristics of the FSI telescope.

\begin{tabular}{|c|c|}
\hline Optical element & Parameters \\
\hline Entrance pupil & Hexagonal, $2.75 \mathrm{~mm}$ edge \\
\hline Occulting disc (OD) & Circular, $\varnothing 8.87 \mathrm{~mm}$ \\
\hline Length Pupil-OD & $135.9 \mathrm{~mm}$ \\
\hline \multicolumn{2}{|l|}{ Mirror (M1) } \\
\hline Figure & Concave ellipsoid $(K=-0.732)$ \\
\hline Dimensions & $\begin{array}{l}66 \mathrm{~mm} \times 66 \mathrm{~mm} \text { substrate } \\
53 \mathrm{~mm} \times 53 \mathrm{~mm} \text { useful area }\end{array}$ \\
\hline Off-axis & $70 \mathrm{~mm}$ \\
\hline Radius of curvature & $925 \mathrm{~mm}$ \\
\hline pass-bands & $17.4 \mathrm{~nm}$ and $30.4 \mathrm{~nm}$ \\
\hline Pupil - M1 centre & $737.35 \mathrm{~mm}$ \\
\hline M1 - Focus & $459.4 \mathrm{~mm}$ along optical axis \\
\hline Filter wheel & Redundant $\mathrm{Al} / \mathrm{Zr} / \mathrm{Al}$ and $\mathrm{Al} / \mathrm{Mg} / \mathrm{Al}$ \\
\hline Detector & Flat, $3072 \times 307210 \mu \mathrm{m}$ pixels \\
\hline Tilt to optical axis & $6.82^{\circ}$ \\
\hline
\end{tabular}

many orders of magnitude more intense than the EUV flux. Placing the entrance pupil in front of the instrument had the added advantage of geometrically reducing the irradiance at the filter centre by a factor of two at perihelion (Auchère et al. 2005).

Classical multi-wavelength EUV telescopes (e.g. SOHO/ EIT, TRACE, STEREO/EUVI, SDO/AIA, Delaboudinière et al. 1995; Handy et al. 1999; Wülser et al. 2007; Lemen et al. 2012) use different multi-layer coatings on different segments of the primary and secondary mirrors combined with a selector mask at the front of the instrument. Since the channels are independent, this design allows complete freedom in the choice of wavebands but multiplies the number of entrance pupils. Given the Solar Orbiter mission profile, FSI instead uses a single entrance pupil in order to minimise the heat load on the instrument ${ }^{8}$. The spectral selection is obtained by a combination of a multi-layer coating reflecting two bands with two types of filters isolating one or the other (see Fig. 6). An $\mathrm{Al} / \mathrm{Zr} / \mathrm{Al}$ ( $52 \mathrm{~nm}$ aluminium/96 $\mathrm{nm}$ zirconium/52 $\mathrm{nm}$ aluminium) filter is used to select the $17.4 \mathrm{~nm}$ pass-band and an $\mathrm{Al} / \mathrm{Mg} / \mathrm{Al}(80 \mathrm{~nm}$ aluminium/322 nm magnesium/80 nm aluminium) filter is used to select the $30.4 \mathrm{~nm}$ pass-band. All filters were procured from the Luxel Corporation. The filter wheel holds two filters of each kind for redundancy against pinholes developing during the lifetime of the instrument. The wheel can potentially also be put in an intermediate position in which the beam is blocked by two mutually exclusive filters. Since there is no shutter in FSI, this position is used when not observing to protect the detector from the EUV-induced degradation suffered by previous EUV telescopes (e.g. EIT, Defise et al. 1999; BenMoussa et al. 2013). Aluminium-based interference coatings have been developed for ten years by Laboratoire Charles Fabry (LCF, Palaiseau, France) in order to achieve higher reflectivity and spectral purity as compared to the EUV coatings used in previous missions (Meltchakov et al. 2010). Moreover, dual-band coatings have been proposed and optimised for FSI in order to reflect two emission lines simultaneously (Auchère et al. 2005; Gautier et al. 2008; Hecquet et al. 2009). The FSI coating consists of a superposition of two periodic multi-layers separated by

\footnotetext{
8 The FSI pupil was initially intended to be positioned at the front of the spacecraft heat shield. The FSI baffle (in front of the aluminium filter) was however not allowed to protrude within the heat shield, requiring a larger aperture in the latter.
}

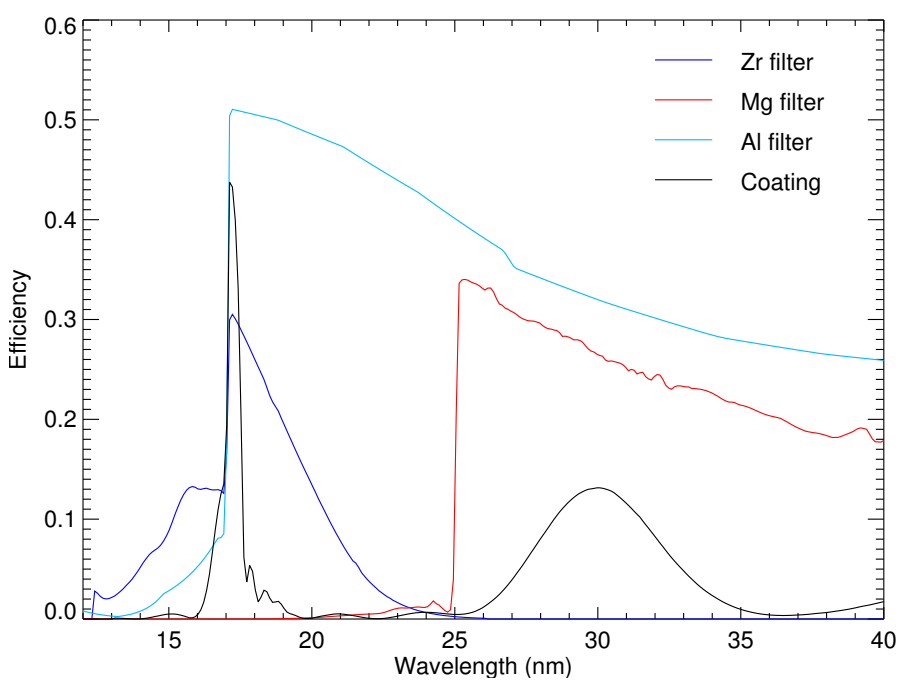

Fig. 6. Spectral selection in FSI. The multi-layer coating (black curve) reflects two bands centred on $17.4 \mathrm{~nm}$ and $30.4 \mathrm{~nm}$. Zirconium (blue curve) and magnesium (red curve) filters are inserted in the beam near the focal plane to select one or the other. The entrance aluminium filter (light blue curve) transmits both bands. Data are from samples of the flight items.

a buffer layer (formula: [SiC/Mo/Al $]^{4} / \mathrm{Al} /[\mathrm{SiC} / \mathrm{Mo} / \mathrm{Al}]^{30}$ ) which is deposited on the mirror substrate. The first structure of the coating is an $\mathrm{Al} / \mathrm{Mo} / \mathrm{SiC}$ periodic multi-layer with first order centred at $17.4 \mathrm{~nm}$ (30 periods of $8.95 \mathrm{~nm})$. An aluminium buffer layer and a second $\mathrm{Al} / \mathrm{Mo} / \mathrm{SiC}$ periodic multi-layer are deposited on top. This second multi-layer is designed to have its first order centred at $30.4 \mathrm{~nm}$ (4 periods of $16.5 \mathrm{~nm}$ ). The outermost $\mathrm{SiC}$ layer is thick enough to guarantee temporal stability so that no additional capping layer is needed. The thickness of the buffer layer is optimised in order to achieve the desired shape of the two Bragg peaks and to create extinctions at undesired wavelengths (Hecquet et al. 2009; Meltchakov et al. 2011, 2013). This enables a spectral purity to be achieved which is comparable to that given by single band coatings in a two-mirror telescope. Ageing, thermal cycling, and exposure to proton radiation have proved these Al-based dual-band coatings to be robust against the Solar Orbiter environment (Delmotte et al. 2013). The resulting pass-bands of FSI are shown in Fig. 23.

Three mirror substrates (FSI1 to FSI3) have been made in Homosil 101, a quartz from the HTM company (formerly Heraeus). These have been hand polished to a sphere and then ion-beam aspherized off-axis by LCF. The measured osculating radii, corrected by the expected $0.128 \mathrm{~mm}$ increase due to the stress induced by the coating (the product stress by thickness was estimated to be 280 Pa.m), were $924.95 \pm 0.04 \mathrm{~mm}$, well within the specified $925.00 \pm 0.3 \mathrm{~mm}$. The effective focal length of the system is $462.5 \mathrm{~mm}$. After coating, the figure errors of the flight model (FSI2) compared to the best conic surface (corresponding to an off-axis distance of $68.89 \mathrm{~mm}$ used for alignment) are $50 \mathrm{~nm}$ peak-to-valley and $7.2 \mathrm{~nm}$ rms (Fig. 7). Given the size and position of the pupil, each field is imaged using $1 \%$ of the mirror's useful area. Therefore, figuring errors on spatial scales greater than the pupil size produce distortions at the focal plane and not degradation of the image quality. Root mean square spot radii are smaller than one pixel across the whole detector. The slope errors do not exceed $5.4 \mu \mathrm{rad}$, which corresponds to the maximum specified distortion of 0.5 pixel at the focal plane. The roughness was measured with a ZYGO5500 over spatial 


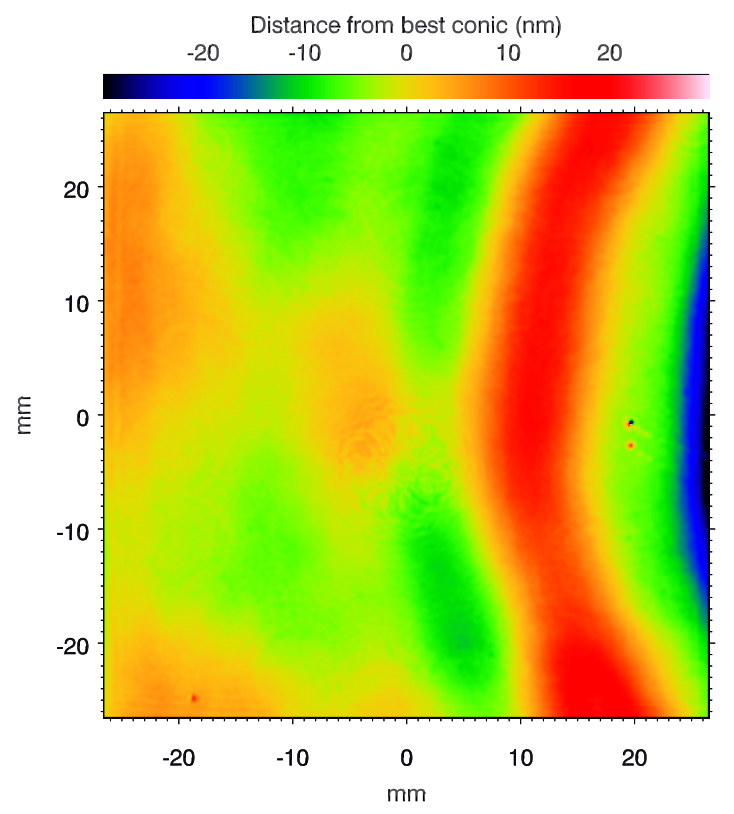

Fig. 7. Figuring errors of the flight model of FSI measured after coating compared to the conic minimising the rms deviations.

frequencies ranging from a few $\mathrm{mm}^{-1}$ to $500 \mathrm{~mm}^{-1}$. The $\mathrm{rms}$ averaged over 14 zones ranges from $0.13 \mathrm{~nm}$ for FSI3 to $0.18 \mathrm{~nm}$ for FSI2. The roughness at the interfaces of the multi-layer coating is $0.5 \mathrm{~nm}$ (Meltchakov et al. 2013).

The coated and mounted mirror is shown in Fig. 8. The combination of a square detector with a small pupil results in an almost square $(53 \mathrm{~mm} \times 53 \mathrm{~mm})$ useful area. The substrate was therefore made square $(66 \mathrm{~mm} \times 66 \mathrm{~mm})$, which represents a $35 \%$ mass reduction compared to the equivalent circular mirror (93 $\mathrm{mm}$ diameter). Additional mass reduction has been achieved (about 50\%, down to $103 \mathrm{~g}$ ) by hollowing the back side.

The small footprint of each field on the optics implies that non-uniformities of the efficiencies translate into local intensity variations at the focal plane. This is equivalent to the pupil of the human eye scanning the lenses of a pair of spectacles. The effect is most pronounced for the focal filters on which the beam footprint is the smallest. This implies that the supporting mesh grid of the focal filter (and to a lesser extent that of the front filter) can potentially produce a stronger modulation in the images than in other EUV imagers. However, as shown in Auchère et al. (2011), the grid modulation is effectively suppressed if one grid period is equal to an integer multiple of the pupil footprint on the focal filter. The grid mesh is thus hexagonal, as the entrance pupil is, with a distance between hexagon centres of $0.4 \mathrm{~mm}$, that is onethird of the beam footprint of $1.2 \mathrm{~mm}$. The grid rods are $0.04 \mathrm{~mm}$ in width, resulting in a uniform geometrical transmission of $0.81 \%$. The focal filters were screened for local defects. The incidence angles on the mirror vary between $88.8^{\circ}$ and $86.3^{\circ}$. This has negligible impact on the uniformity of the spectral response of the multi-layer coating across the mirror. Thickness variations of the coating produce a $0.1 \mathrm{~nm}$ shift of the pass-bands towards the long wavelengths from the centre to the corners of the FOV.

Following the descope of the $30.4 \mathrm{~mm}$ channel of Metis, FSI became the only instrument on Solar Orbiter able to explore the extended corona in the EUV up to 10 solar radii, an as yet largely unexplored territory. The FSI was however not originally designed to be able to observe up to these heights. Stray light in EUV imagers can dominate the signal at large angles from

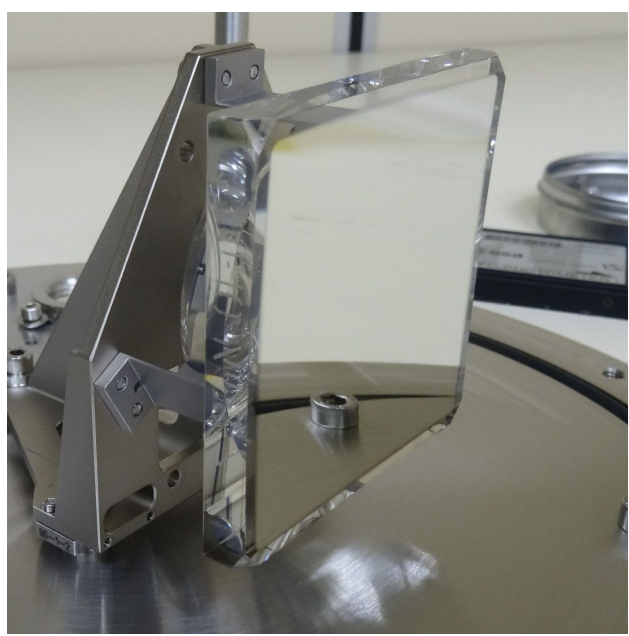

Fig. 8. FSI mirror coated and bonded on its mount.

the solar limb (e.g. Auchère \& Artzner 2004) due to a combination of diffraction by the entrance mesh grid and scattering off the optical surfaces. Late in the development it was therefore decided that the capability of blocking direct solar disc light would be added by adapting a knife-edge occulting disc on the door mechanism in front of the entrance filter. The computed integrated intensity in the shadow cast on the mirror is $6 \times 10^{-3}$ that of the solar disc at $30.4 \mathrm{~nm}$ and $4 \times 10^{-3}$ of the solar disc at $17.4 \mathrm{~nm}$. It is therefore expected that the stray-light levels will be decreased by the same factors compared to previous, unocculted EUV imaging telescopes, although performance at the working wavelength could not be verified due to the very low expected scattering levels. With a vignetting cutoff at $0.777^{\circ}$, the occulting disc is designed to be used at distances from the Sun larger than $0.34 \mathrm{AU}$, that is, during the remote sensing windows nominally centred on the highest solar latitude points of the orbits. At shorter distances, the stray-light level is expected to be sufficiently low in the whole FOV to operate without the occulting disc. The vignetting function increases to 0.49 at the edge and 0.75 in the corners of the FOV. The cutoff corresponds to varying distances to the solar limb along the orbit. The FSI door was qualified for 200 open-close operations, which allows for several FSI coronagraphic campaigns per orbit throughout the mission lifetime. The occulting disc will also be used to characterise the stray light by comparison with regular data.

\subsection{High Resolution Imagers}

The two HRI units share a similar two-mirror optical design (Fig. 5). The HRI Lya channel (Schühle et al. 2011) is an offaxis Gregory telescope, and the HRI $\mathrm{EUV}_{\mathrm{EV}}$ channel (Halain et al. 2015) is an off-axis Cassegrain telescope optimised in length and width. The entrance pupils are located at the front section of dedicated entrance baffles and have a diameter of $47.4 \mathrm{~mm}$ for the $\mathrm{HRI}_{\mathrm{EUV}}$ and $30 \mathrm{~mm}$ diameter for the $\mathrm{HRI}_{\text {Lya }}$.

The Gregory design of the $\mathrm{HRI}_{\text {Lya }}$ channel allows the use of a field stop in the light path which, combined with internal baffle walls, strongly limits the stray light from out of the FOV. The HRI $\mathrm{EUV}_{\mathrm{V}}$ channel is made of a Cassegrain telescope which allows for better mirror positioning tolerancing but has less stray-light reduction capabilities (given the absence of an intermediate focus). The optical prescription of the two HRI channels is given in Table 2 . 
Table 2. Optical design parameters of the HRI $\mathrm{EUV}_{\mathrm{EV}}$ and $\mathrm{HRI}_{\mathrm{Lya}}$ channels.

\begin{tabular}{lll}
\hline \hline & HRI $_{\text {EUV }}$ & HRI $_{\text {Lya }}$ \\
\hline Focal length & $4187 \mathrm{~mm}$ & $5804 \mathrm{~mm}$ \\
Entrance pupil & $47.4 \mathrm{~mm}$ & $30 \mathrm{~mm}$ \\
Field of view & $1000 \mathrm{arcsec}^{2}$ & $1000 \mathrm{arcsec}^{2}$ \\
Plate scale & $50 \mathrm{arcsec} \mathrm{mm}^{-1}$ & $31.5 \mathrm{arcsec} \mathrm{mm}^{-1}$ \\
Detector & $2048 \times 2048,10 \mu \mathrm{m}$ pixels & $2048 \times 2048,14.1 \mu$ m virtual pixel size \\
Primary mirror (M1) & $66 \mathrm{~mm} \emptyset(54 \mathrm{~mm}$ useful $) 80 \mathrm{~mm}$ off-axis & $42 \mathrm{~mm} \emptyset(38 \mathrm{~mm}$ useful $), 80 \mathrm{~mm}$ off-axis \\
& $\mathrm{RC}=1518.067 \mathrm{~mm} \mathrm{CC}=-1$ & $\mathrm{RC}=1143 \mathrm{~mm} \mathrm{CC}=-1$ \\
Secondary mirror $(\mathrm{M} 2)$ & $25 \mathrm{~mm} \emptyset(12 \mathrm{~mm}$ useful $), 11.44 \mathrm{~mm}$ off-axis & $20 \mathrm{~mm} \emptyset(18 \mathrm{~mm}$ useful $), 7$ mm off-axis \\
& $\mathrm{RC}=256.774 \mathrm{~mm} \mathrm{CC}=-2.04$ & $\mathrm{RC}=91 \mathrm{~mm} \mathrm{CC}=-0.65$ \\
\hline
\end{tabular}

Notes. Here, $\mathrm{RC}$ is the radius of curvature and $\mathrm{CC}$ is the conic constant.

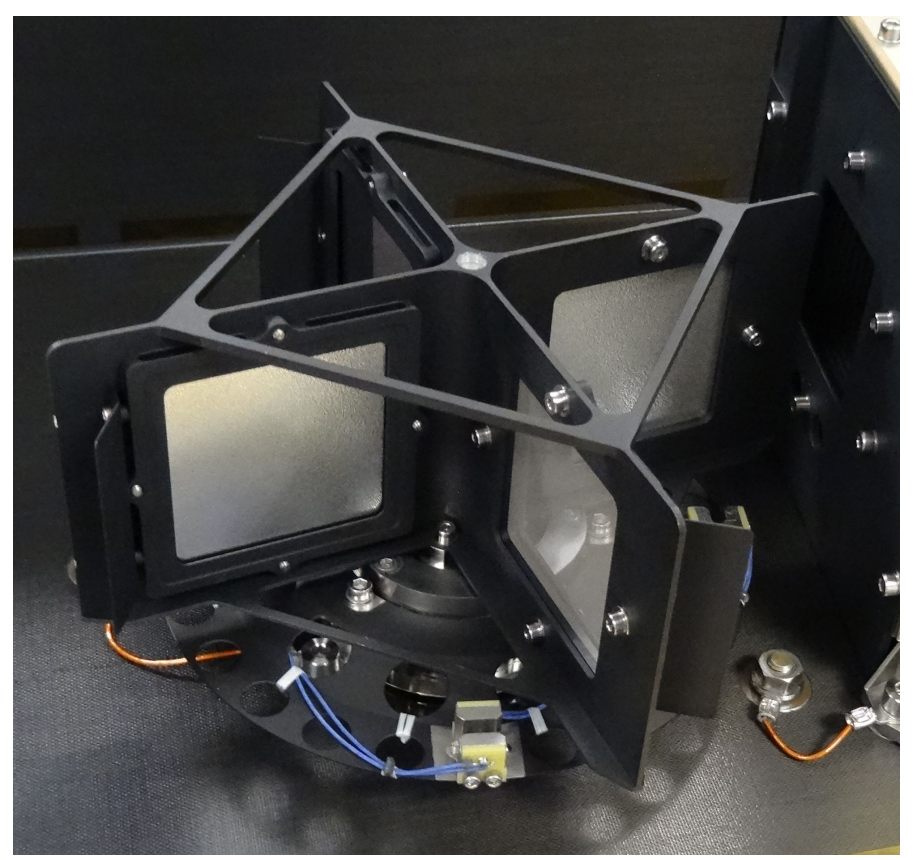

Fig. 9. Filter wheel of FSI integrated on the OBS.

The EUV multi-layer coatings of the mirrors are specifically optimised to provide the optimal spectral pass-bands. Their design takes into account the angle of incidence on the mirrors, the variations of which are small enough so that no compensation is needed. Aluminium-based EUV interference coatings have been developed specifically for HRI $\mathrm{EUV}_{\mathrm{V}}$ by LCF. Identical coatings have been deposited on both primary and secondary mirrors. The variations of the angle of incidence between primary and secondary and on each mirror are small enough so that no compensation in period thickness is needed. Periodic $\mathrm{Al} / \mathrm{Mo} / \mathrm{SiC}$ multi-layer coating with first order centred at $17.4 \mathrm{~nm}$ has been specifically optimised to provide high efficiency and desired spectral pass-bands. This consists of 30 periods with $8.86 \mathrm{~nm}$ period thickness. A $3 \mathrm{~nm} \mathrm{SiC} \mathrm{capping} \mathrm{layer} \mathrm{was} \mathrm{deposited} \mathrm{on} \mathrm{top}$ in order to improve temporal stability. Aging, thermal cycling, and exposure to proton radiation have proved these periodic $\mathrm{Al} / \mathrm{Mo} / \mathrm{SiC}$ multi-layer coatings to be robust against the Solar Orbiter environment (Delmotte et al. 2013). These HRI EUv passband filters were adapted to the wavelengths with respect to the out-of-band suppression and the spectral purity of the pass-band. In Fig. 10 we show the figuring errors of the flight model of the

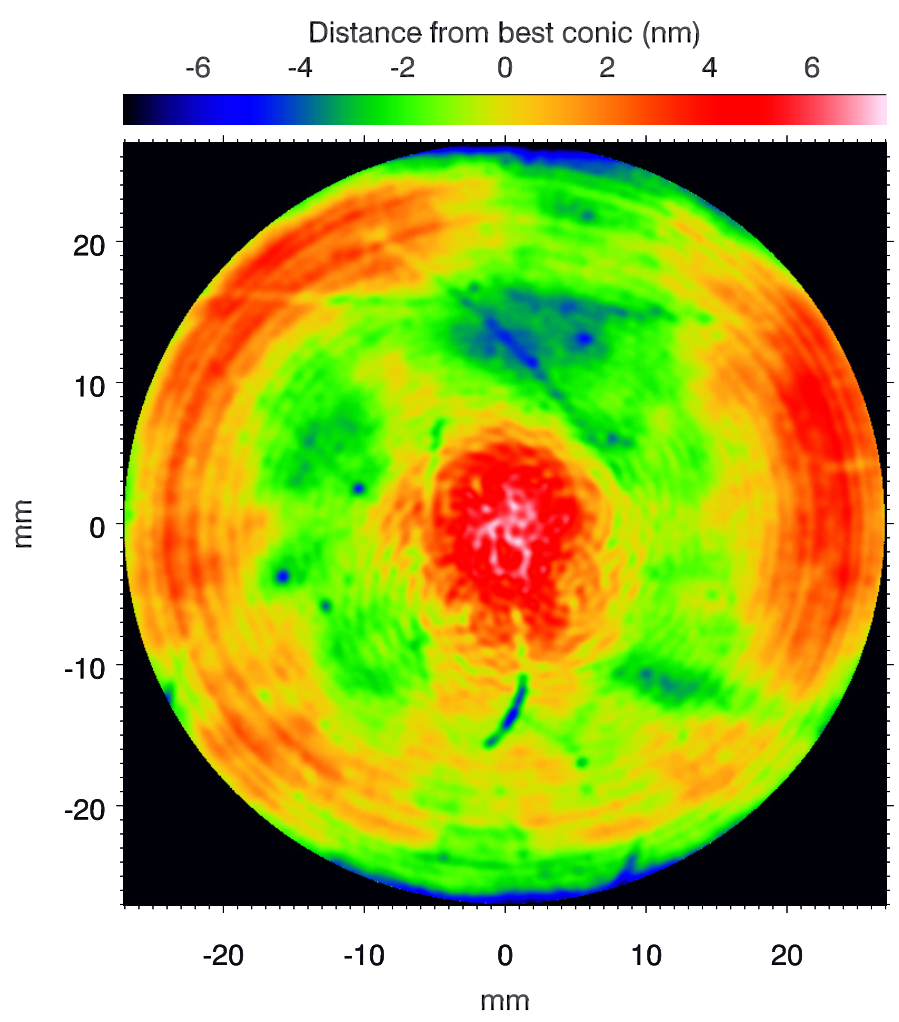

Fig. 10. Figuring errors of the flight model of the HRI $\mathrm{E}_{\mathrm{EUv}}$ primary mirror measured after coating compared to the conic minimising the rms deviations. Ringing is an artefact on the measurement setup.

$\mathrm{HRI}_{\text {EUV }}$ primary mirror measured after coating compared to the conic minimising the rms deviations.

For the HRI EUV channel, one aluminium foil filter is inserted between the entrance aperture (entrance pupil) and the primary mirror to provide protection against excessive heat input on the mirror and efficient rejection of the visible light. This HRI $\mathrm{IUV}_{\mathrm{EUV}}$ entrance filter is a standard filter from Luxel Corporation supported by a nickel mesh grid of 20 lines per inch (lpi) reinforced with a rib structure dividing the filter area over quadrants to improve thermal conductivity and mechanical resistance. A filter wheel with four filter slots is located at the exit pupil. These four slots each contain two $150 \mathrm{~nm}$ aluminium filters (nominal and redundant) from Luxel Corporation, with a 20 lpi nickel grid support, one open slot (no filter), and one occulting slot. The HRI filter wheel, as in the FSI one, is qualified for a million operations. Similarly to the FSI channel, the HRI $\mathrm{EUV}_{\mathrm{EV}}$ front and rear EUV filters avoid contamination with visible light. 


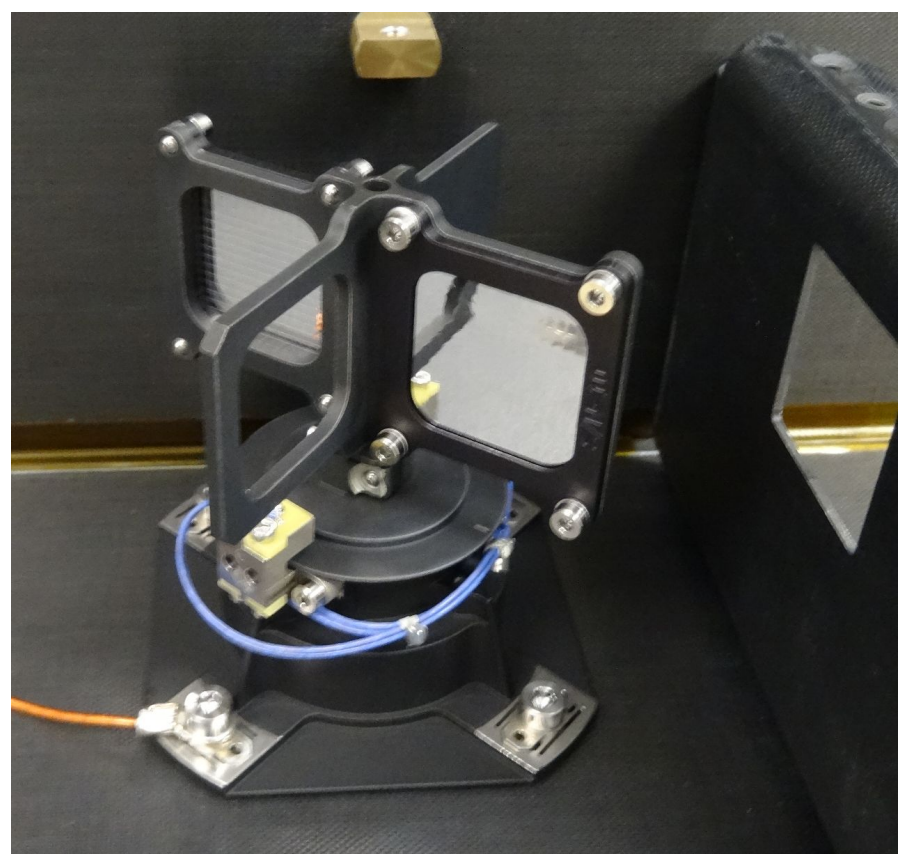

Fig. 11. Filter wheel of HRI integrated on the OBS.

The $\mathrm{HRI}_{\text {Lya }}$ mirrors use $\mathrm{Al} / \mathrm{MgF}_{2}$ (aluminium/magnesium fluoride) coatings providing a reflectivity at $121.6 \mathrm{~nm}$ of over $86 \%$. A broad-band interference filter (Type 122 NB-40D of Pelham Research Optical LLC) is used at the entrance of the telescope to isolate the spectral line at $121.6 \mathrm{~nm}$ and reject visible and infrared light as well as EUV and X-rays to protect the mirror coatings. A narrow-band filter (Type $122 \mathrm{XN}-2 \mathrm{D}$ of Acton Research Co.) is placed in front of the detector to further isolate the Lyman- $\alpha$ line and achieve the spectral purity. The combination with a solar-blind detector yields a spectral purity higher than $90 \%$ for Lyman- $\alpha$ in the quiet Sun regions. It is tolerant to spectral shifts potentially caused by thermal effects. The magnesium fluoride substrate material of the filters is sufficiently radiation hard so as to provide protection against the thermal heat load with minimal degradation during the mission.

\section{Mechanical and thermal design}

The EUI is a compact instrument based on a passive thermomechanical design meaning that there is no active thermal control, but there is passive detector cooling. To avoid contamination which would degrade the instrument response, the EUV sensitive detectors of the EUI instrument must however never be the coldest parts of EUI, and in particular its focal plane assemblies (FPAs). In nominal flight conditions, this is ensured by a welldesigned FPA, including a "cold cup" that is slightly colder than the detectors to trap contaminants, and by the presence of heaters for annealing and evaporating condensate accumulated on the surface of the detectors. The instrument contains one nominal heater per detector to allow in-flight bake-out (annealing) and a thermal sensor, as part of the detector packaging. When a heater is in use, the corresponding camera electronics are powered off. These heaters will be used sequentially (not together) and controlled by the CEB following a configurable duty-cycle to ensure the temperature of the detectors does not exceed the maximum non-operating (NOP) temperature. These decontamination/annealing heaters are $84.3 \Omega$ each, which at $28 \mathrm{~V}$ is $9.3 \mathrm{~W}$ per camera with a separate control on each. Because the heaters use the spacecraft $28 \mathrm{~V}$ power, the heat output may vary as the voltage varies.

Before nominal flight conditions, that is, just after spacecraft launch, the instrument will not be powered on for possibly several hours. To limit the temperature drop of the detectors during venting of the instrument and spacecraft out-gassing, it is therefore planned to heat the three EUI detectors via a second set of spacecraft-controlled "post-launch" heaters controlled by a thermostat and located on the path between each detector and the two cold element (CE) interfaces (see Figs. 3 and 16). As soon as possible after launch, these heaters are powered by the spacecraft and remain on as long as possible until commissioning. These post-launch heaters are three series of $15 \Omega$ heaters totalling $17.4 \mathrm{~W}$ for the EUI OBS.

\subsection{Common Electronics Box}

The CEB is radiatively and conductively coupled with the spacecraft structure (Fig. 3). The CEB housing is made of aluminium alloy, has a volume of approximately $0.007 \mathrm{~m}^{3}$, and contains four printed circuit boards (PCBs: a Compression Board, a Processor Board, a power supply board, and an auxiliary board) that are mechanically and thermally coupled with the CEB housing. The functions of the four main boards of the CEB are described below in Sect. 5. The CEB box (Fig. 2) has a base plate with six M6 screws for attachment to the spacecraft, and a coating of Aeroglaze Z306 which was chosen for its thermal and reflectance properties. The CEB has nine connectors for the EUI internal harness (data, control and power transfer from CEB to OBS) and four connectors for the spacecraft harness (nominal and redundant power and data interfaces).

\subsection{Optical Box}

The OBS unit structural housing is composed of sandwich panels (CFRP - Carbon Fibre Reinforced Polymer facesheets/aluminium honeycomb core) to support the optical elements with the required stiffness and thermal stability while achieving a low mass. The EUI design has been adapted to the mass, volume, and thermal constraints imposed by the spacecraft. The OBS unit is supported by three titanium A-shape mounts providing thermal decoupling and an isostatic mechanical interface with the spacecraft mounting panel. The main bench is a panel of $30 \mathrm{~mm}$ in thickness with reinforced honeycomb at the interface with the three mounts. The back panel is $20 \mathrm{~mm}$ thick and holds the two primary mirrors of the HRI channels. The other OBS panels (internal walls between telescopes, lateral and front panels) are $10 \mathrm{~mm}$ thick, but only contribute to the OBS unit structural stiffness. Under the OBS unit, the harness from the electrical subsystems (camera and mechanisms) is routed toward a connector panel at the rear side.

All optical subsystems, either primary mirror or secondary mirror, filter wheel, or FPA, have their separate interfaces. This arrangement allows for clearly defined interfaces with a minimum in thermo-elastic deformation of each channel, providing, in particular, mirror inter-distance and telescope co-alignment stability. Internal baffles made of CFRP layers are also implemented within the HRI telescopes to limit potential stray light from the entrance to the detectors. A top cover (10 mm thick) provides additional torsional and bending stiffness to the overall structure.

In contrast to the CEB, the OBS is a thermally insulated unit (Fig. 3) that remains in an acceptable temperature range in hot 
and cold flight environments. It has a low conductive link to the platform (low conductance mounts) and radiative insulation (two-layer MLI), taking advantage of the optical bench with a low coefficient of thermal expansion (CTE) on which the three EUI telescopes are mounted and co-aligned. The OBS is an internally mounted instrument within the spacecraft cavity.

The thermal design of the EUI OBS unit takes into account the specific thermal environment of the Solar Orbiter mission and in particular of the 13 solar constants solar heat flux $\left(17.5 \mathrm{~kW} \mathrm{~m}^{-2}\right)$ at perihelion. The front of the OBS unit (doors and entrance baffles) is consequently thermally decoupled from the rest of the OBS unit to limit thermo-elastic deformation and ensure optical performance over the operational temperature range of $-20^{\circ} \mathrm{C}$ to $+50^{\circ} \mathrm{C}$. It is however thermally coupled with the spacecraft by two redundant heat pipes to evacuate the solar and infrared heat absorbed by the entrance baffles and filters (see Fig. 3).

Being directly exposed to the highly varying solar flux, the ultra-thin EUV front filters are subject to large variations and spatial gradients. An optimised custom frame was developed that divides the exposed area into smaller cells without significantly altering the optical throughput. In this way, the temperature gradient could be reduced by more than a factor of two. The EUI detectors must be cooled to a temperature lower than $-40{ }^{\circ} \mathrm{C}$ (target $-60^{\circ} \mathrm{C}$ ) with a stability of $\pm 5^{\circ} \mathrm{C}$. The OBS unit is also therefore thermally coupled to some spacecraft radiators through dedicated thermal straps, allowing the transfer of dissipated heat by the detectors (through a cold element (CE) interface) and by the camera electronics boards (medium element (ME) interface); see Fig. 3. Because of spacecraft constraints, the two HRIs share a common $\mathrm{CE}$ and common ME interface with the spacecraft, while the FSI has its own CE and ME interfaces.

\subsection{Filter wheels}

The EUI employs two filter wheels, one in FSI and the other one in HRI EUv. Both have been developed by IAS with support from the Centre National d'Etudes Spatiales. The FSI filter wheel (Fig. 9) is used for wavelength selection and is therefore a critical component that has been qualified for approximately 1 million operations (quarter turns), corresponding to a worst case of continuous observations at two wavelengths at $10 \mathrm{~min}$ cadence through the end of extended mission phase, and onground operations, with a safety factor of 1.2. The HRI $\mathrm{EUV}_{\mathrm{V}}$ filter wheel (Fig. 11) on the other hand provides redundancy for the focal filter and a masking position to protect the detector against radiation-induced degradation and was designed for 400 operations. Due to mass and volume constraints, mechanisms are of the paddle wheel type. The mass distribution of the different elements was precision balanced in order to avoid the need for a launch-lock system. The design also minimises the thermoelastic stresses with the optical bench. The filter wheels are connected to the rotor of their respective motor via elastic couplings without gear reduction stages. The axis of the FSI wheel is supported by two ball bearings dry-lubricated with $\mathrm{MoS}_{2}$. Due to its light weight, the HRI wheel is directly supported by the bearing of its motor. Both motors are custom dual-winding steppers from Phytron $\mathrm{GmbH}$. The temperatures of the motors are controlled by thermal sensors in the motors driven by the CEB.

Cancellation of the grid modulation pattern in FSI (see Sect. 3.1 and Auchère et al. 2011) requires positioning of the filters to within $\pm 0.5 \mathrm{~mm}$ along the beam and a rotational accuracy of $\pm 1^{\circ}$. This latter requirement was a major design driver. The stepper motor has 200 steps, and additional margin is obtained using micro-stepping control. The whole mechanism was aligned to bring its reference position to within $\pm 0.17^{\circ}$ of its nominal orientation with respect to the optical axis $\left(8.66^{\circ}\right)$. The lifetime test has demonstrated the repeatability of the behaviour. The knowledge of the FSI filter wheel position is ensured by a combination of four opto-isolators.

\section{Electronics}

All the EUI electronics are grouped in the CEB (Sect. 5.2), except for the so-called Front End Electronics (FEE) which are associated with the sensors in the three telescope cameras (Sect. 5.1).

\subsection{Cameras and sensors}

Each telescope has its own dedicated camera (also called Focal Plane Assembly) mounted on the OBS main bench. The EUI cameras are based on large-format $3072 \times 307210 \mu \mathrm{m}$ pitch CMOS-APS detectors, allowing shutterless operation, greater radiation tolerance (no charge transfer), low power consumption, high operational temperature $\left(>-60^{\circ} \mathrm{C}\right)$, high speed, high dynamic range, and non-destructive readout. The devices consist of two stitching blocks of $3072 \times 1536$ pixels and are based on the architecture shown in Fig. 12. In order to reach ambitious goals for both the read noise and the dynamic range, a dual-gain pixel design was developed: each pixel outputs both a high-gain signal, with low read noise and a low saturation level, and a lowgain signal, with a high saturation level but larger read-noise. The sensor is controlled through 32 register settings that control, among others, gain factors, clipping values, and offset values. The detectors have a full well of the order of 120k electrons (device dependent), which is the same for both gains (defined by saturation of the pinned photodiode), but the high gain is ADC clipped. The theoretical ratio in gain between the high and low gain channels with default electronic settings is 22.3 . In practise, this ratio is device dependent and configurable in flight.

The EUI detector package is designed to take into account the thermo-mechanical constraints of the camera; see Fig. 13. It is made of aluminium nitride (AlN) providing sufficient thermal conductivity and similar CTE to that of the sensor and is supported by three Ti blades providing thermal decoupling from the mounting interface. A copper strap is used to provide the thermal link to the spacecraft radiators (Fig. 14). The electrical connection to the FEE is achieved through a flexible PCB. The temperature is monitored with a thermal sensor integrated into the detector. For decontamination purposes, the detector is also equipped with a heater allowing heating and in-flight bake-out sequences to be performed if required.

The $\mathrm{HRI}_{\text {Lya }}$ camera detector is a solar-blind, intensified, CMOS active pixel sensor (I-APS). The camera intensifier is a closed unit held under vacuum by a $\mathrm{MgF}_{2}$ entrance window and a fibre optic output window. The output window is glued to a fibre-optic taper, which is coupled with the image sensor by direct contact with the active area of the sensor and mechanically stabilised by epoxy gluing with an Invar frame to the sensor package. The intensifier also has a blocking filter such that it transmits no visible light and only light produced by electrons from the micro-channel plate (MCP) will be detected by the CMOS sensor.

The intensifier of $\mathrm{HRI}_{\mathrm{Lya}}$ was built by ProxiVision $\mathrm{GmbH}$ (Germany) and shares the same design as the Metis UV detector (Schühle et al. 2018): a closed tube with a UV-grade magnesium fluoride entrance window and a single MCP that transforms 


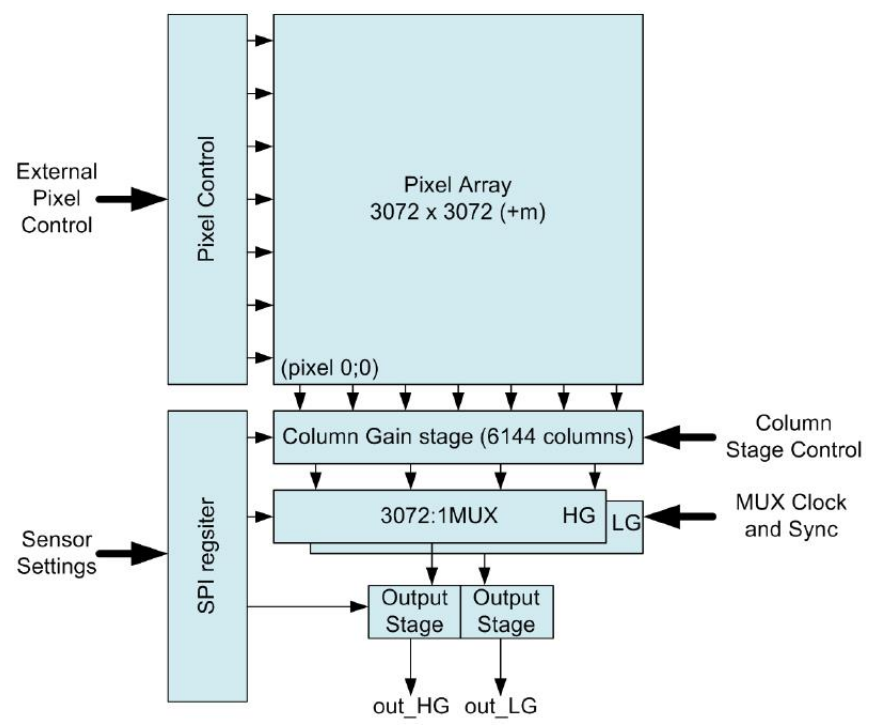

Fig. 12. EUI $3072 \times 3072$ detector architecture block diagram.

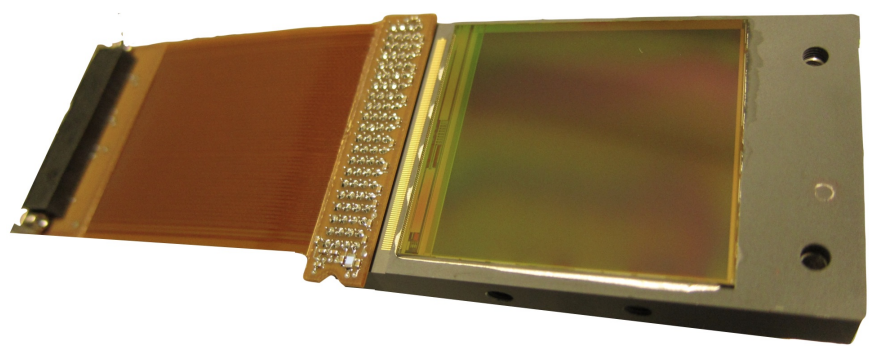

Fig. 13. EUI flight detector with package and connector via flexible PCB.

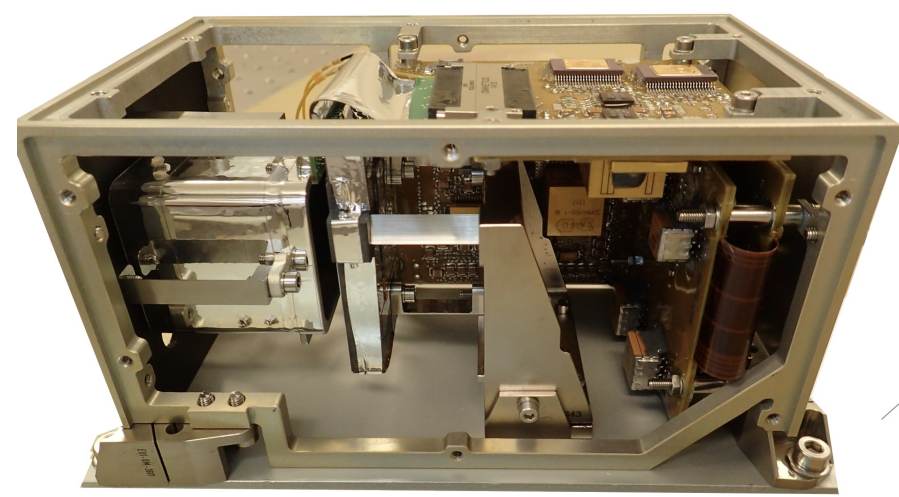

Fig. 14. $\mathrm{HRI}_{\mathrm{EUV}}$ camera flight model. See also Fig. 16 for a schematic layout.

the incoming UV photons into electrons which are accelerated against a phosphorous screen. The focal plane of the camera coincides with the entrance face of the MCP where the photocathode is deposited. The photocathode of potassium bromide $(\mathrm{KBr})$ provides high sensitivity at $121.6 \mathrm{~nm}$ and suppresses the response at longer wavelengths. The photo-electrons amplified by the MCP are accelerated by a high voltage (of nominally $6 \mathrm{kV}$ ) onto a $\mathrm{P} 46$ phosphorous screen. A regulated high voltage unit (HVU) provides the voltages for the MCP and the phosphorous screen. The sensitive aperture of the intensifier tube is $40 \mathrm{~mm}$ in diameter, and a square image of $28.3 \mathrm{~mm} \times 28.3 \mathrm{~mm}$ on the phosphorous screen is transferred by a fibre optic face

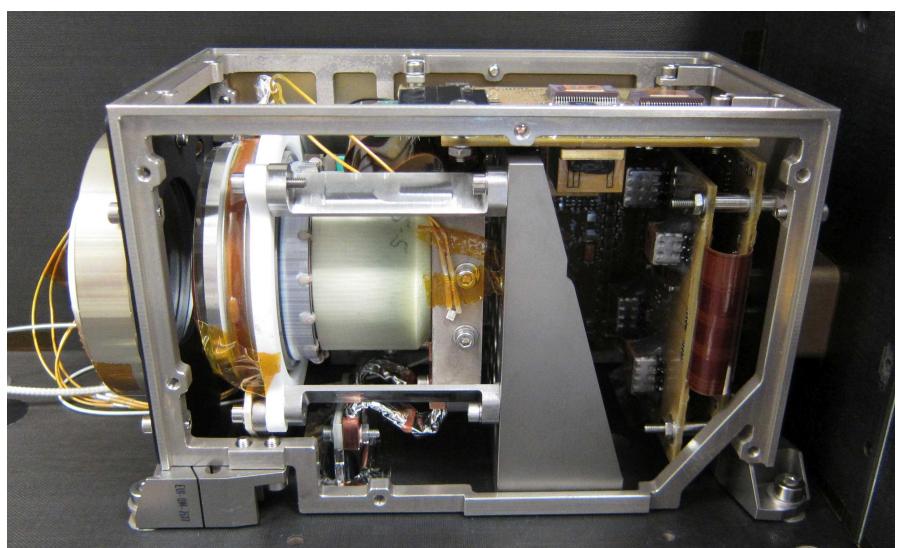

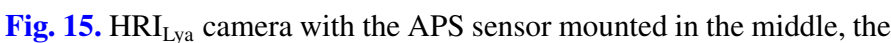
Front End Electronics (FEE) on the right hand side, and the intensifier on the left hand side. See also Fig. 16 for a schematic layout.

plate and a fibre optic taper with a de-magnification factor of 1.41 to the actual size of the CMOS-APS sensor. In this way, the image size is reduced to cover only a $2048 \times 2048$ subarea on the $3072 \times 307210 \mu \mathrm{m}$ pixel sensor. This provides an image scale of the telescope of 1 arcsec per two pixels. Due to the mechanical mounting of the fibre optic image transfer, the useful size of the taper on the sensor is slightly smaller than 2048 pixels (actual size is $1984 \times 1984$ pixels), corresponding to a FOV of 992 arcsec $\times 992$ arcsec. The intensifier assembly has the advantage of having a very low sensitivity to radiation above $150 \mathrm{~nm}$ (because of the photocathode response) and below $115 \mathrm{~nm}$ (because of the $\mathrm{MgF}_{2}$ window), thus greatly helping to isolate the wavelength of interest. The intensifier is mounted with a ceramic ring via four blades to the camera housing, while the APS sensor is hard-glued to it and connected only by thermal straps to the CE interface (Fig. 15).

The HRI EUV and FSI channels share a common FPA design, as shown in Fig. 16 (top). A cold cup is located in front of the detector and is linked to the same cooling path as the detector in such a way as to ensure the detector is not the coldest part of the instrument to reduce condensation of molecular contamination on the detector. The cameras also include two redundant UV LEDs (at $255 \mathrm{~nm}$ ) for ground tests and in-flight relative calibration mounted in such a way that they obliquely illuminate the whole detector.

The HRI Lya $_{\text {a }}$ channel camera uses the same FPA mechanical design as the HRI $\mathrm{EUV}_{\mathrm{EU}}$ and FSI channels but it carries the intensifier unit (see bottom of Fig. 16). The HRI Lya camera does not have a cold cup, as the detector is directly glued to the intensifier, and its detector is maintained in position through the intensifier fixation to the structure.

The intensifier tube requires regulated high voltages up to $1 \mathrm{kV}$ and $6 \mathrm{kV}$ maximum for the MCP and the phosphorous screen. An external HVU switches an isolated secondary power to obtain these voltages up to a maximum of $7 \mathrm{kV}$. Differential analogue lines from the CEB control the two high voltage (HV) outputs. Differential analogue outputs are fed back to the CEB to monitor the HVU outputs. The HVU provides HV panel connectors on the backside of the housing. Pigtail cables for the screen and the MCP are soldered on the intensifier side and plugged to the panel connectors on the HVU. The low voltage (LV) electronics part is separated on the $\mathrm{PCB}^{9}$ from the $\mathrm{HV}$ parts with appropriate spacing (assuming a maximum permitted DC

\footnotetext{
9 Printed Circuit Board.
} 

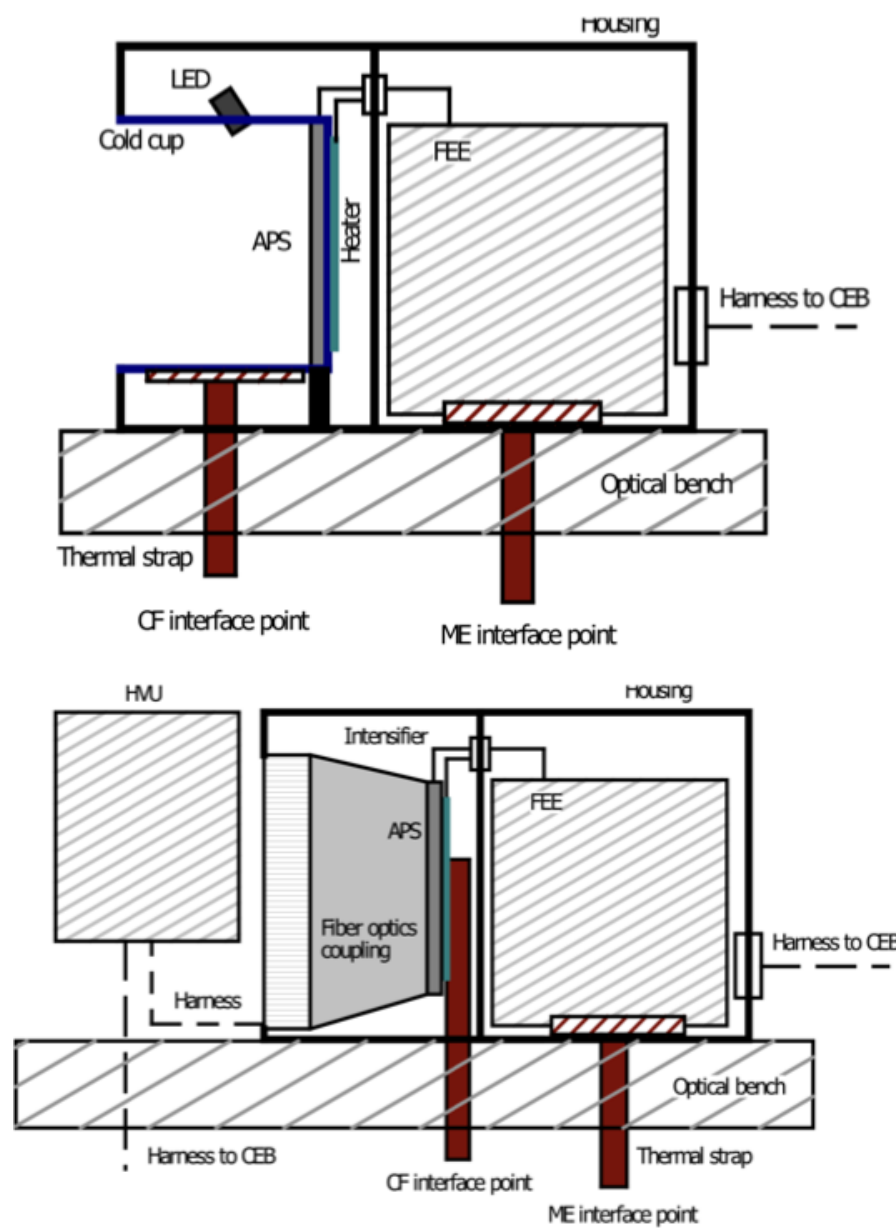

Fig. 16. Camera layout for FSI and HRI $I_{\mathrm{EUV}}$ cameras (top) and for $\mathrm{HRI}_{\text {Lya }}$ camera (bottom). Photons enter the camera from the left and reach the CMOS APS from where the signal is read-out to the FEE and ultimately to the CEB (right). The $\mathrm{HRI}_{\text {Lya }}$ camera (bottom) has an additional HVU and intensifier. In brown, the Cold Finger (CF) thermal strap and ME thermal strap are shown.

voltage of $600 \mathrm{~V} \mathrm{~mm}^{-1}$ across surfaces) to prevent discharges or a propagation of $\mathrm{HV}$ to the $\mathrm{LV}$ parts. The HV circuitry is put on the top layer of the PCB and no traces from the LV side are routed within or below this area. High voltage tracks are routed with rounded edges to prevent strong electrical fields on edges. Primary and secondary ground on the HV converter are completely separated on the PCB and connected together only at a single ground point at the secondary output side of the HV transformer. This single ground point is routed to the Structure GND (ground) via a (removable) ground stud connection. The outputs for screen and MCP are floating at the connected intensifier side and grounded at the converter output. The HVU is mounted separately from the camera inside the $\mathrm{HRI}_{\mathrm{Lya}}$ channel on the optical bench.

\subsection{Common Electronics Box}

The CEB electronics consist of four PCBs: the Processor Board, the Compression Board, the Auxiliary Board, and the Power Board. These are all plugged into a motherboard (which has no components other than the connectors for the other boards). The interfaces to the Solar Orbiter Spacecraft are prime and redundant $28 \mathrm{~V}$ power connections, prime and redundant SpaceWire connections and high-power pulse command connections for

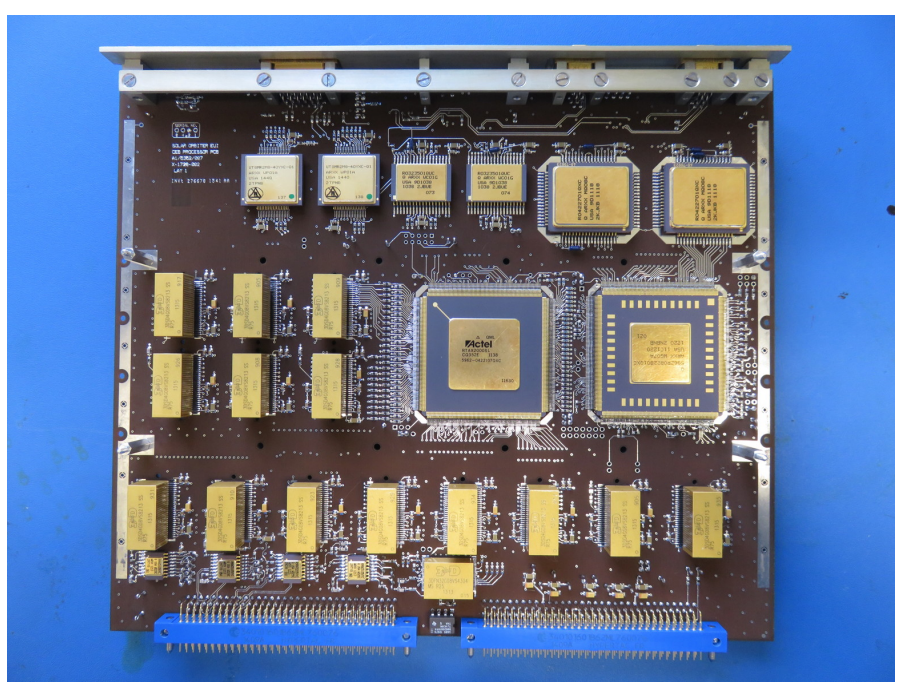

Fig. 17. Processor Board in the EUI CEB. At the bottom of the board there are gigabytes of SDRAM for the One-Hour-Queue (OHQ) seen as eight blocks, and at the very bottom in the middle is the flash memory. The Spacecraft Output Buffer (SOB) is located in the middle left as six blocks. The Processor Board FPGA is at the centre with the UT699 CPU to the right. The two components to the top left are the MRAM. The next four components (two small, two large) on the top right are the CPU SRAM.

commanding power on and power off. The SpaceWire links allow telecommands to be sent to the CEB and telemetry (housekeeping, events, and science data) to be sent from the CEB. Other CEB interfaces connect with the OBS to provide power to the cameras, heaters, and mechanisms to allow data to and from the cameras and output from thermistors and thermocouples on the OBS.

\subsubsection{The Processor Board}

The Processor Board contains the CEB's Central Processing Unit (CPU), a Cobham-Gaisler single UT699 LEON3 SPARC Application-Specific Integrated Circuit (ASIC) which also has four SpaceWire interfaces, two of which are used. The CPU is connected to some write-disabled Magnetoresistive RandomAccess Memory (MRAM) and some read/write MRAM from which to boot the Basic "Boot software" and Oper "Application software", some Static Random-Access Memory (SRAM) as main processor memory and to an Actel Field-Programmable Gate Array (FPGA). This Processor Board FPGA provides an interface to gigabytes of Synchronous Dynamic Random-Access Memory (SDRAM) for immediate and longer-term storage of science data (the One Hour Queue (OHQ) and Spacecraft Output Buffer (SOB)), the usage of which is described in Sect. 6.3. The FPGA also provides an interface to flash memory for nonvolatile storage of calibration data as well as interfaces to the other FPGAs of the CEB on the other boards. Figure 17 shows the Processor Board.

\subsubsection{The Compression Board}

The Compression Board consists of an FPGA which has some fast SRAM for storage of pixel-processing maps, a WICOM compression ASIC with SDRAM to perform fast wavelet data compression, and the interfaces for the incoming data from the cameras. Figure 18 shows the Compression Board. As data 


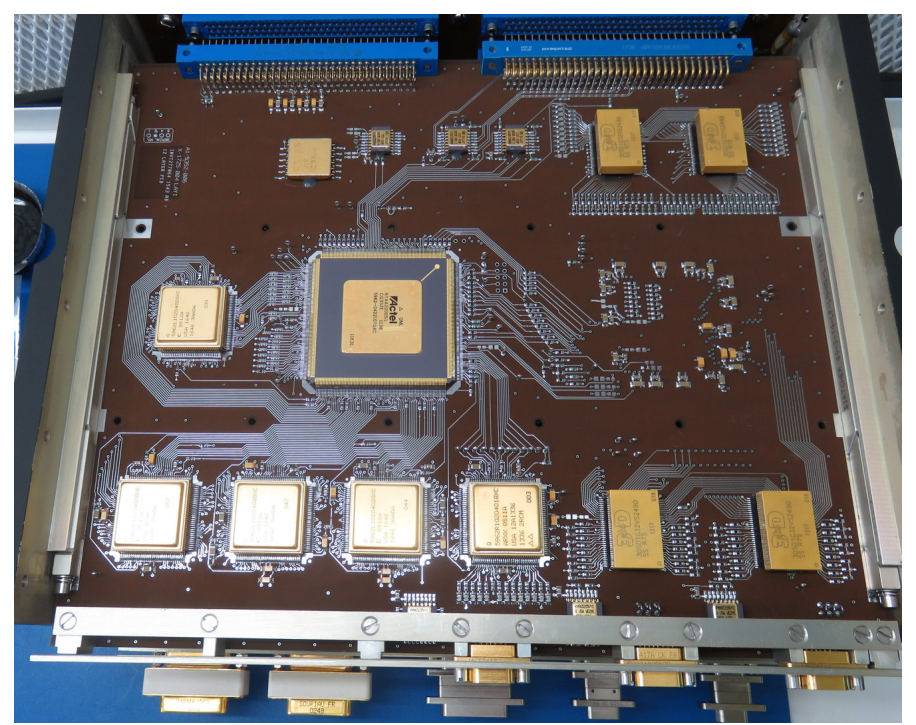

Fig. 18. EUI CEB Compression Board. The Compression Board FPGA is the large integrated circuit near the centre. The block on the middle left together with the first three of the bottom row are the compression SRAM. The WICOM is the fourth block of the bottom row. On the top right and bottom right there are four SDRAMs for the WICOM.

arrives from a camera, various stages of on-the-fly image processing can be enabled. The aim of this onboard image processing is to enhance the effectiveness of data compression (discontinuities produced by detector features would degrade the ability of the compression). These stages operate on an imageby-image basis and are: per-pixel and global gain correction, perpixel and global offset correction, bad pixel correction, cosmic ray filter, rebinning, thumbnail and histogram generation, and recoding (Nicula et al. 2005).

As the detectors have two different gains in dual-gain mode, strongly exposed pixels (bright) will report a low gain value while weakly exposed pixels (dark) will report a high gain value. The images arriving at the compression board will thus contain a mixture of pixel values of both of the gains. As a result, the FPGA is required to apply a global gain and offset correction (which are simple adjustable constants) and a per-pixel gain and offset correction which are pre-loaded in the compression SRAM as a pair of maps, one for each of the two gains.

The SRAM maps also contain maps of bad pixels. A pixel is labelled as "bad" when its sensitivy or dark current deviates significantly from that of its neighbours. The value of a bad pixel is replaced as it arrives at the FPGA with the value of the last good pixel in the image. If the bad pixel is the first pixel in a line, then the value of the first pixel in the previous line is used, and for the first pixel of the first line, an adjustable constant is used. The cosmic ray filter identifies significant point-like outliers originating from both cosmic rays and solar energetic particles, and replaces them with a local $3 \times 3$ median-like value which is calculated not by processing the image as a whole but by buffering recent lines and working as the pixels arrive.

For very high compression ratios (lower bits per pixel), it is beneficial to bin the image before compression. This can be enabled in the FPGA with a choice of $2 \times 2$ and $4 \times 4$ binning. This reduces the effective spatial resolution but provides an overall better image quality for applications when the ultimate spatial resolution is not required. As the pixels arrive, the FPGA can also calculate the image histogram and a highly binned thumbnail before the image is sent for compression, after which the software can no longer "see" the original image. The histograms are intended to retain minimal information on images that cannot be brought to the ground.

The final optional stage of pixel processing is recoding in which each incoming pixel is scaled between user-defined low and high values and the integer square root ( 8 bit) is calculated.

Once the pixel processing has made the image suitable for compression, if enabled, the image is sent to the WICOM for compression. An ASIC such as the WICOM is required as the compression must be performed very quickly (half a second) during the time that the next image is being integrated on the detector (which could be a half-second exposure). As many images are taken and only the relevant ones (e.g. with solar activity) are selected, the compression speed and power efficiency of an ASIC solution is essential for the speed and power constraints of EUI.

\subsubsection{High-Speed Links}

The CEB communicates with the cameras by pairs of HighSpeed Links (HSL), one pair for each camera, which are based on SpaceWire but simplified with a different protocol. Two HSLs were used per camera to enhance the speed of data transfer. The efficient design allows simple short commands to be sent from the $\mathrm{CEB}$ to the cameras at a slower rate, whereas the responses to commands and images from the cameras to the CEB are at a fast configurable rate to deal with data volume.

\subsubsection{The WICOM}

The WICOM ASIC was developed for the PLEIADES mission by EADS-Astrium, now Airbus. It uses a JPEG2000-like wavelet compression called MRCPB which is optimised for use in space missions and can perform lossless or lossy compression at a wide range of bit rates. The WICOM uses dedicated SDRAM for temporary storage. The EUI benefits from WICOM versatility including a compression mode where a large image of $3072 \times 3072$ (or square subfields thereof, as for the HRI $2048 \times 2048$ images) is compressed globally at very high compression ratio (less than 0.1 bits per pixel with the aid of binning and recoding), automatically allocating the few bits to describe the most important local events in the solar disc image.

\subsubsection{The Auxiliary Board}

The Auxiliary Board contains an FPGA which drives the stepper motors of the filter wheels and doors, gathers analogue and digital parameters from the CEB, and controls and monitors the electronic current trips and the power switching (heater control, door launch locks and camera power). Figure 19 shows a photograph of the auxiliary board.

\subsection{The Power Board}

The Power Board supplies isolated secondary power for the CEB and the three cameras. It is made up of four switching DC/DC isolating Power Supply Units (PSUs), one for the CEB and one for each of the cameras. This design allows the number of switches to be minimised by turning the PSUs of the three cameras on and off at their inputs rather than at their more numerous outputs. The Power Board interfaces to the spacecraft prime and redundant power buses. Figure 20 shows a photo of the Power Board. 


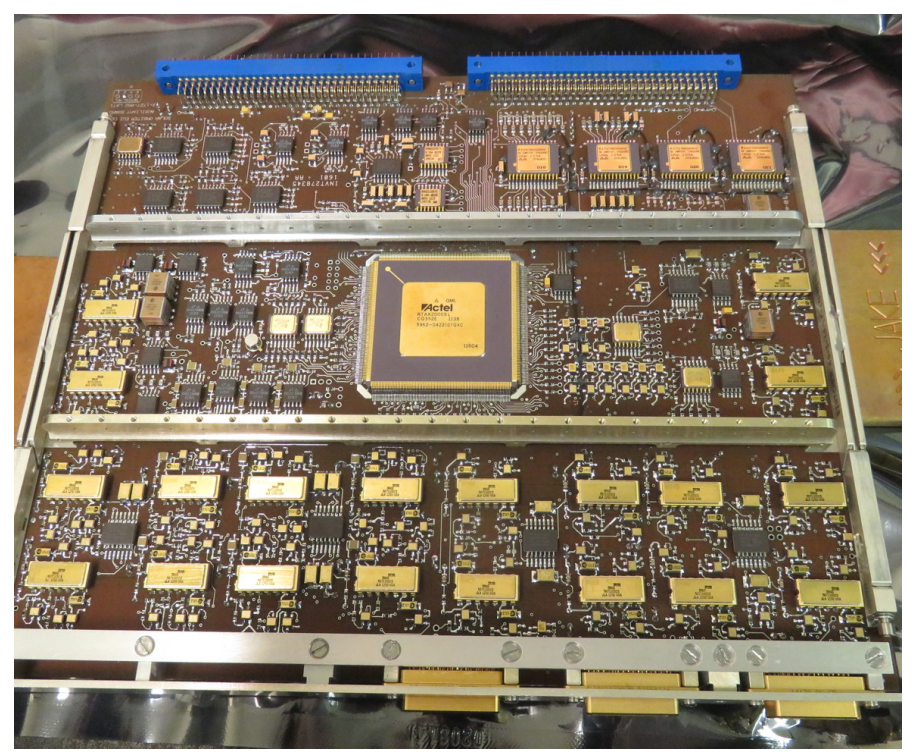

Fig. 19. EUI CEB auxiliary board. The AUX board FPGA is at the centre. The bottom third of the board contains the motor drive circuitry. Housekeeping is digitized in the top part of the board.

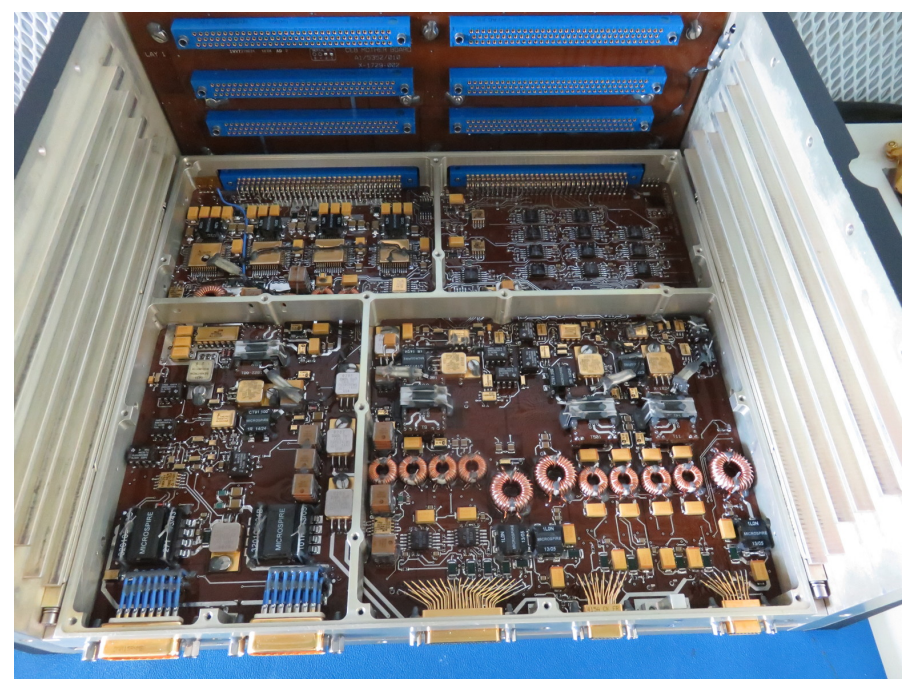

Fig. 20. EUI CEB power supply unit. The top left are the voltage regulators for internal supplies, the top right is the housekeeping filtering and conditioning, the bottom left is the CEB PSU, and the bottom right are the camera PSUs.

The electronic parts are qualified for space use and are suitable for the high radiation environment outside the Earth's magnetosphere and near the Sun. Some of the memories have to be continually checked and corrected for errors ("scrubbed"). This is done for some devices by the device itself, for some by an FPGA, and for others by the software. It is expected that the flash memory will develop bad blocks throughout its lifetime, which will be dealt with by the software.

\section{Flight software}

\subsection{Software overview}

The EUI flight software runs on the Cobham-Gaisler LEON SPARC $^{10}$ processor of the CEB. It uses the Gaisler-modified

\footnotetext{
${ }_{10}$ Scalable Processor Architecture.
}

RTEMS $^{11}-4.10 .2$, a well-written, robust, and well-maintained real-time open source operating system. Software development is performed on Linux with GNU tools. With the source code available for RTEMS, the compilation tools and Linux, this offers the highest level of maintainability for a long mission.

The software consists of two separate subsystems. Basic (boot) software starts on boot-up and is as simple as possible yet able to load and dump memory and perform health monitoring and reporting. This code is stored in read-only non-volatile memory. In contrast, the Oper (application) code supports full functionality and is stored separately in re-writable non-volatile memory so that it may be patched after launch.

Compliance to ECSS (European Cooperation for Space Standardisation) and MISRA (Motor Industry Software Reliability Association) was strictly maintained during development with very few exceptions. Thus, an extensive test programme and defensive programming makes the software very robust and maintainable as is necessary for a space mission.

RTEMS is used as the interface to the CPU which also contains the SpaceWire interfaces. It was modified only slightly to meet the necessary functionality. RTEMS provides the scheduler, interrupt handling, tasks (threads), semaphores, and message queues that are required by the software in a manner which is familiar to the programmer.

The software is written in a highly modular fashion with modules well separated and tasks communicating with each other using RTEMS message queues for good synchronisation and safety.

\subsection{Basic software functionality}

Incoming telecommands are received by the Gaisler RTEMS SpaceWire driver and interrupts are generated. Two telecommand tasks process these telecommands (one task for each of the redundant interfaces). The required checks are performed on the telecommands and they are passed on to the appropriate modules of the software to be checked and processed further. If requested in the telecommand, verification acceptance and completion telemetry packets are generated and any errors produce verification failure packets.

Approximately 2000 housekeeping parameters are sent to the ground periodically in nine different housekeeping telemetry packets to report the state and health of the instrument. This is handled by the housekeeping module with its task. Memory management is handled by its module and task with the ability to load, dump, and checksum all memories where possible. Memory scrubbing and watchdog management is performed by another module with its task. The memories that are to be scrubbed by software are continuously read and checked, and single bit errors are corrected. If multiple bit errors are found, either an error will be sent or the system will be rebooted depending on the affected memory. Event reporting is performed by its module to allow the ground and spacecraft to keep track of normal progress, warnings, and errors. The testcommand module simply provides the ability to respond to a test command to check connectivity. Another module, timesync, updates and reads the clock in the CEB's Processor Board FPGA and timestamps packets as required. The functionality described above is common to Basic and Oper.

${ }_{11}$ Real-Time Executive for Multiprocessor Systems. 


\subsection{Higher software functionality and science}

Both Basic and Oper are able to receive inter-instrument communication (IIC), also referred to as inter-instrument messaging (IIM) telecommands. These are discarded by Basic whereas they are acted upon, if enabled, by Oper. These telecomands are sent from the spacecraft but composed of data not only concerning the spacecraft but also sent by the instruments. They contain information on spacecraft status and the observing status and "interesting events" found by the instruments. Science telemetry packets are produced only by Oper and contain images, histograms, metadata, and calibration products.

The flight software operates the three cameras, setting up various registers, reading back responses from each command, and then commanding an exposure. As the cameras respond to commands and can send messages unrequested, an elaborate state machine makes sure that the command and response protocol is maintained in a robust manner with error detection, retries, and recovery.

Exposures are sequenced by the sequencer task in the sequencer module, which reads observing tables (discussed below) and received start- and stop-sequence telecommands. The resulting image data are received by the compression FPGA of the CEB which performs selectable pixel pre-processing before passing on the data to the WICOM compression ASIC if requested. The compressed data from the WICOM are stored temporarily and sequentially in the One Hour Queue (OHQ). Normally, the sequencer then requests that the data are transferred from the OHQ to the Spacecraft Output Buffer (SOB) for long-term storage with error detection and correction (EDAC) against bit-flips from radiation. As the pixel pre-processing and compression are done "on the fly" as the pixels arrive, the software cannot access full images, however image "thumbnails" (highly binned images) and histograms can be requested for the images, and the software can process these to check for quality and interesting events (see Sect. 9.5).

\section{Cleanliness and contamination control}

\subsection{Cleanliness requirements}

Contamination of any kind may degrade the performance of a $\mathrm{UV}$ instrument by reducing the efficiency of the telescope and detection system and by reducing contrast due to increased stray light BenMoussa et al. (2013). The purpose of contamination control is to maintain the scientific performance of the instrument. All possible contaminants affecting the performance have been identified in the cleanliness requirements specification document. The EUI cleanliness requirements have been derived by estimation of the expected degradation of performance caused by particulate or absorbing molecular matter. As a UV instrument, the EUI channels are particularly sensitive to organic contamination, which is strongly absorbing within the used scientific wavelength ranges. Sources of such contamination are mainly outgassing products of unavoidable organic compounds used for instrument construction. For this reason, the materials used for EUI structures and subsystems have been selected according to and scrutinized for their contamination potential. In addition, many features have been implemented in the design of the instrument to minimise contamination of the optical system. The contamination limits have been estimated for the EUI instrument in terms of the maximum tolerable loss of throughput and scattered light. The requirements are dictated by the maximum acceptable degradation of performance in terms of contrast, throughput, and dynamic range of the telescopes and the detection systems. As
Table 3. Maximum limits of tolerable contamination at any time of the mission.

\begin{tabular}{lll}
\hline \hline Sensitive Area & Molecular $\left(\mathrm{ng} \mathrm{cm}^{-2}\right)$ & Particulate $(\mathrm{ppm})$ \\
\hline Entrance baffle & 370 & 300 \\
Internal surfaces & 370 & 100 \\
External surfaces & 500 & 300 \\
\hline
\end{tabular}

a working assumption, an overall loss of throughput of $50 \%$, either by scattering or absorption, was considered tolerable. We further assumed a uniform distribution of contaminants in each telescope channel. The sensitive surfaces are the optical components of the three channels: entrance and focal filters, mirrors, and detection system. The coverage by contaminants was allocated to six optical surfaces, resulting in $7 \%$ loss of throughput per surface due to coverage. As a worst-case assumption we have assumed that particulate contamination coverage is opaque and molecular contaminants are absorbing at the scientific wavelength range. The contamination limits determined in this way are summarized in Table 3.

\subsection{Contamination control}

To stay within the contamination limits, the control program consisted of the general strategy: material selection, precision cleaning of parts, vacuum baking at parts level, integration of subsystems in cleanrooms of Class ISO-5, and final bake-out of the EUI instrument before delivery to the spacecraft. In addition, important contamination control features have been implemented by design:

- The instrument was equipped with three entrance door mechanisms that allow opening and closing of the optical path independently for each channel. Rather than one-shot doors, the EUI doors are closable, to protect the optical system during nonoperational phases. The doors serve many purposes: they protect against contamination entering the optical path during ground operations; limit the ultraviolet and solar wind irradiation on the first optical element during non-operational phases, while the Heat Shield doors remain open during most of the time of the mission; reflect (in closed position) the heat flux from the Sun in the same way as the front filter, to minimize thermal disturbance between door operations; limit the air flux during ground operations (and acoustic loads during launch) around the fragile entrance EUV filters. To fit behind the entrance baffle, close to the entrance filter, the doors have been specially designed in the form of a sliding door with a mechanism that rotates the door lid into its closed position where it is blocking the aperture not tightly but with a labyrinth opening around the lid circumference.

- The structural housing, which is made of lightweight CFRP panels, has no other openings than the labyrinth seal of the doors and two venting holes per channel in the bottom side of the optical bench. All panels have small holes for venting outward from the structure.

- A purge line distribution system has been installed that supplies clean purge gas to the interior of each channel. Vertical walls separate each channel with a seal between all panels such that each channel has its own compartment with a purge inlet and purge outlet. Flow restrictions at the purge inlets define the relative flow rate supplied to each channel. Venting holes serve as purge outlet with flow restrictions to avoid sudden pressure changes during ground activities that might cause structural damage to the foil filters of the two EUV channels. 
These cleanliness-related activities have been specified in the Contamination Control Plan. A contamination budget was established to allocate the amount of possible contamination during each phase of ground activities until delivery. The particulate contamination occurs only during ground activities by exposure to the cleanroom environment, while molecular contamination continues during vacuum testing and the operational phase in space. The instrument was assembled in a cleanroom environment (Class ISO-5) and once finished it was kept under purging with nitrogen. When the purging had to be interrupted for short phases during the environmental test campaign the instrument was covered with bagging foil. Finally, before delivery of the instrument for integration on the spacecraft, a thermal-vacuum test was performed, followed by a bake-out at $80^{\circ} \mathrm{C}$. The process was monitored in real time with a temperature-controlled quartz crystal microbalance (TQCM) held at $-20^{\circ} \mathrm{C}$, and the bake-out was successfully terminated when the change of the mass deposition rate on the TQCM was less than $1 \%$ for at least $3 \mathrm{~h}$.

\section{Performance as designed}

The EUI instrument requirements were derived from the EUI observations that are needed to address each of the four overarching Solar Orbiter Science questions (see Sect. 2). These EUI instrument requirements were the basis of the instrument design discussed in the previous sections. Verification of these requirements was done through performance characterisation of all optical elements at subsystem level (detectors, filters, mirrors) and finally through end-to-end calibration of the flight unit.

Given the obvious science impact, particular attention was given to the verification of the relative spectral response of each telescope and, given the small telescope apertures, to the absolute throughput. In this section we report the performance as designed and illustrate this with a few preliminary calibration results, while the full end-to-end calibration of each telescope will be the subject of dedicated papers.

\subsection{Absolute response}

Imaging at high resolution (and hence high cadence to avoid dynamic blurring) with small-aperture telescopes requires high throughput and low noise levels. Faint coronal features such as disturbances in loops (flows or waves) or eruptions (off-limb, EIT waves) are typically at $10 \%$ of the background intensity (Robbrecht et al. 2001; Thompson \& Myers 2009). This imposes a minimum $\mathrm{S} / \mathrm{N}$ of 10 , with a minimum signal to be detected of 2 photons per pixel.

This translated to particularly severe requirements on the telescope sensors. A very low read noise was required on the high-gain readout $\left(<5 \mathrm{e}^{-} \mathrm{rms}\right)$ and the dark signal was required to be smaller than $10 \mathrm{e}^{-} \mathrm{pix}^{-1} \mathrm{~s}^{-1}$ at $-40^{\circ} \mathrm{C}$. During its development, the detector spatial noise was lowered, as confirmed by early measurements with qualification and flight detectors. Fixed pattern noise (FPN, pixel offsets) may be further suppressed using onboard dark-frame correction. Given the low dark current at cold detector temperature (typically $-30{ }^{\circ} \mathrm{C}$ ), the only remaining non-uniform noise is the photo-response non-uniformity (PRNU). The detector quantum efficiency, or sensitivity, at $17.4 \mathrm{~nm}$, defined as the ratio between captured and incident photons, was confirmed to be superior to $80 \%$ during calibration measurements. The detectors are designed so that MTF at Nyquist frequency shall be above $50 \%$ at working wavelength $(17.4 \mathrm{~nm})$. Their crosstalk at working wavelength shall

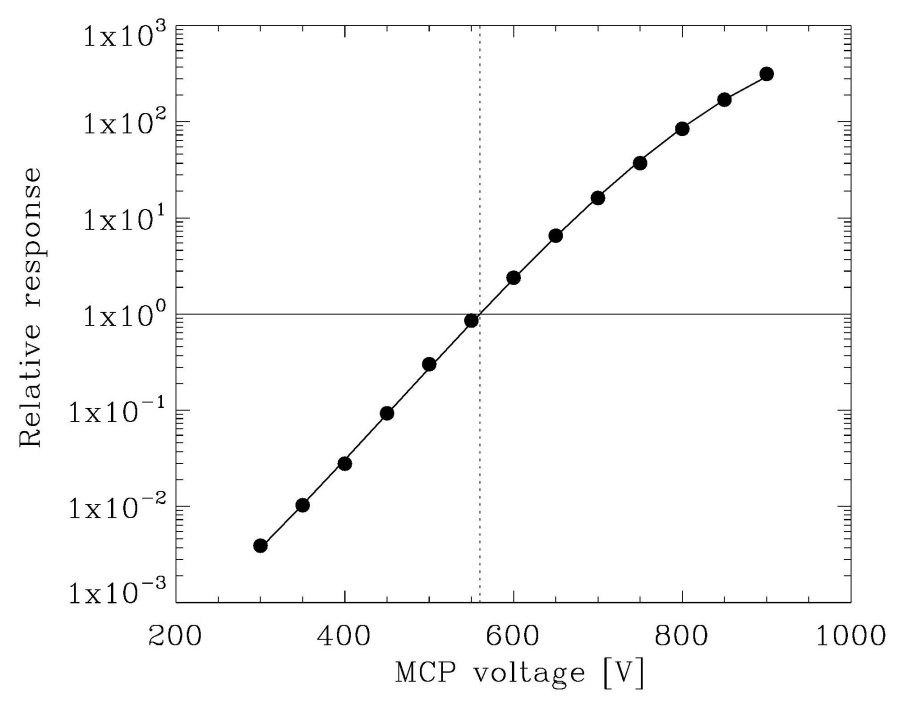

Fig. 21. Relative response of the $\mathrm{HRI}_{\mathrm{Lya}}$ camera vs. voltage applied to the MCP of the intensifier normalised at $560 \mathrm{~V}$.

be below 5\%. Bad pixels and cosmic rays are removed before onboard image and lossy compression (see Sect. 5.2.2).

The response of the Lyman- $\alpha$ camera is particularly dependent on the voltage applied to the micro-channel plate of the intensifier. This was characterised in a series of measurements at MPS using a monochromatic Lyman- $\alpha$ light source. As we show in Fig. 21, the response of the intensifier can be regulated by MCP voltage between $300 \mathrm{~V}$ and $900 \mathrm{~V}$ and within this range the voltage can be selected to stay in the linear dynamic range of the sensor. The variation of the gain is linear on a logarithmic scale between $400 \mathrm{~V}$ and $750 \mathrm{~V}$. This gives a relative gain increase of approximately an order of magnitude per $100 \mathrm{~V}$.

\subsection{Relative spectral response}

Because of the rise of the solar spectrum at long wavelengths, integration of off-band radiation over a large spectral range can result in significant contamination of the total signal. The spectral response of the instrument subsystems (detectors, filters, mirrors) have therefore been characterised, not only at the spectral band of interest, but also over a very large wavelength range beyond that.

For $\mathrm{HRI}_{\mathrm{EUV}}$, the baseline was a spectral purity greater than $90 \%$ for two subsequent ionisation states for Fe IX $(17.11 \mathrm{~nm})$ and $\mathrm{Fe} X(17.45 \mathrm{~nm}$ and $17.72 \mathrm{~nm})$, when observing quiet Sun and active region. In Fig. 22 we show the expected spectral response of $\mathrm{HRI}_{\mathrm{EUV}}$ based on simulated mirror-coating data, measured entrance-filter transmission, and measured detector QE.

For FSI, the single bounce inherently provides less off-band rejection than two-bounce designs like that of HRI $\mathrm{EUV}_{\mathrm{EU}}$. Whenever possible, the FSI optical components have therefore been measured from 10 to $100 \mathrm{~nm}$. The FSI was designed to be sensitive to local sensitivity variations of the optics (Sect. 3.1). The spectral response was specified to be uniform to within $5 \%$ on each component.

For $\mathrm{HRI}_{\text {Lya }}$, because of the broad-band reflectance of the mirrors, two interference filters were necessary in addition to the solar blind detector to achieve the desired spectral purity: an entrance filter with $20 \mathrm{~nm}$ FWHM bandwidth and $20 \%$ peak transmission, and a narrow-band focal plane filter with $10 \mathrm{~nm}$ bandwidth and $8 \%$ peak transmission. All components have been 


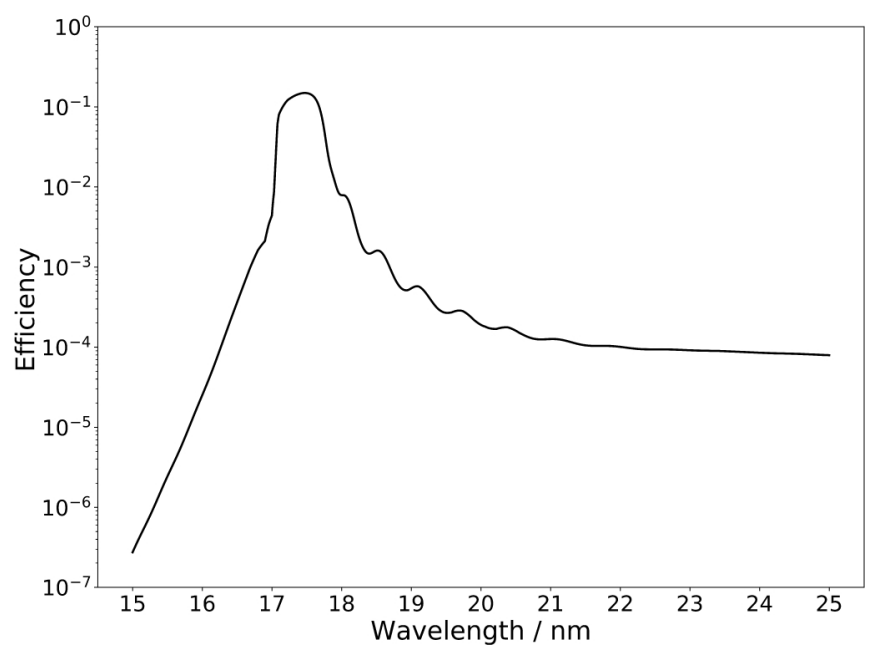

Fig. 22. Expected spectral response of $\mathrm{HRI}_{\mathrm{EUV}}$, assuming rear filter in open position. A detailed description can be found in Gissot \& EUI consortium partners (in prep.).

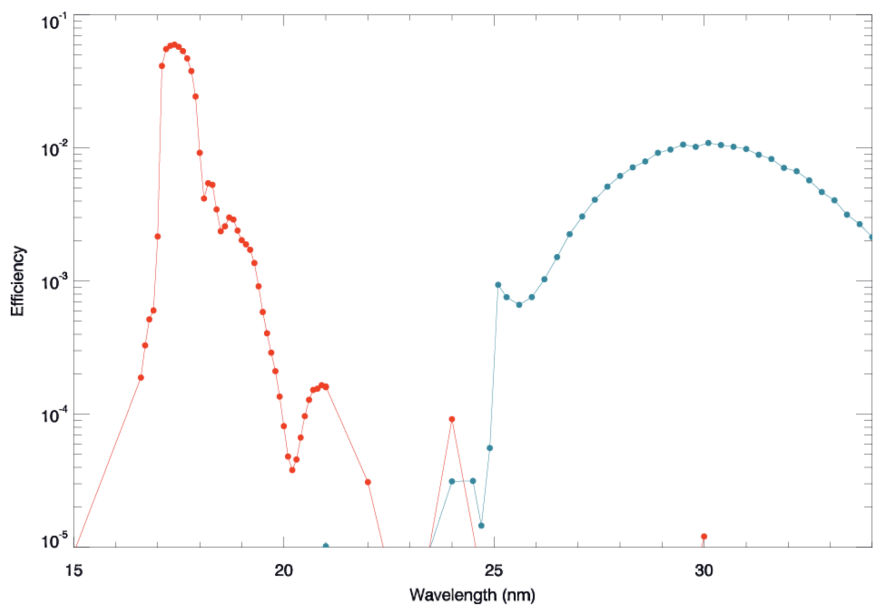

Fig. 23. Relative spectral response of the EUI/FSI channel from 15 to $34 \mathrm{~nm}$, showing the $17.4 \mathrm{~nm}$ channel in red and the $30.4 \mathrm{~nm}$ channel in blue. A detailed description can be found in Auchère \& EUI consortium partners (in prep.).

characterised individually for their in-band and out-of-band performance before integration into the instrument.

To further suppress the signal longward of Lyman- $\alpha$, the image intensifier uses a multichannel plate with a potassium bromide photocathode. For stabilisation of the MCP gain the intensifier was irradiated by a Lyman- $\alpha$ lamp for about two weeks. In order to characterise the long-wavelength suppression of the strong solar continuum, which is mainly achieved by the $\mathrm{KBr}$ photocathode deposited on the MCP, the camera was calibrated at PTB over a large wavelength range, from $115 \mathrm{~nm}$, limited by the cutoff of the magnesium fluoride, up to the signal-to-noise limitation above $300 \mathrm{~nm}$. The spectral calibration of the camera was performed with the MPC at a voltage of $400 \mathrm{~V}$. The response of the camera at any other setting of the MCP voltage can be obtained by scaling with the gain function shown in Fig. 21 .

In Fig. 24, we show the relative spectral response predicted by the measurements of the components between $115 \mathrm{~nm}$ and $300 \mathrm{~nm}$. We note that the peak of the response (solid line in the figure) is shifted by $2.6 \mathrm{~nm}$ with respect to the Lyman- $\alpha$ line as a result of the narrow-band focal plane filter maximum

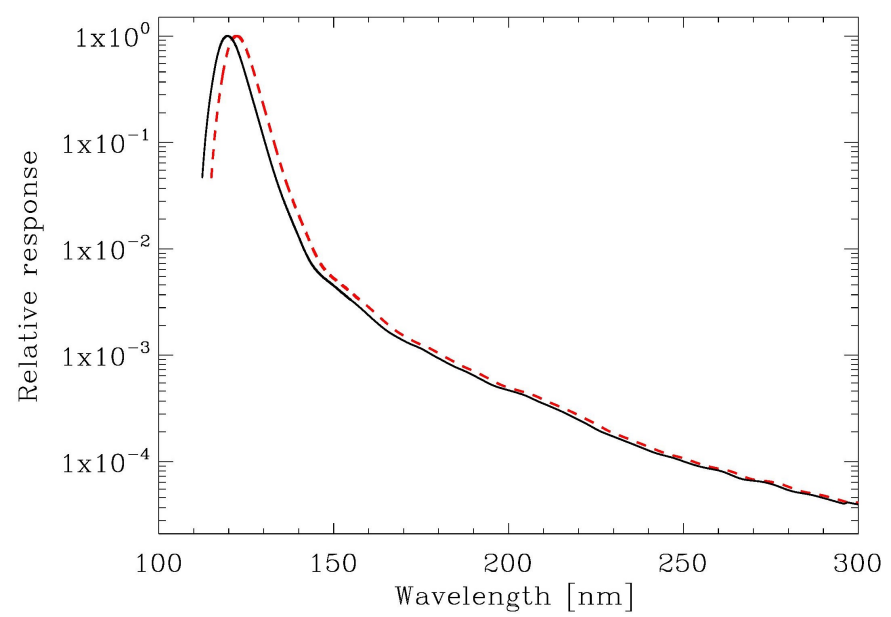

Fig. 24. Relative spectral response of $\mathrm{HRI}_{\mathrm{Lya}}$ as predicted by the measured (black solid) and the theoretical efficiencies of the components (red dashed). A detailed description can be found in Schühle \& EUI consortium partners (in prep.).

transmittance at $119 \mathrm{~nm}$. The dashed line corresponds to the relative response calculated with efficiencies from literature values while the solid curve has been shifted to coincide with the measured data.

\subsection{End-to-end calibration}

End-to-end radiometric measurements were performed at the Metrology Light Source (MLS) of the Physikalisch-Technische Bundesanstalt (PTB) in Berlin. The electron storage ring provides calibrated radiation at the desired wavelengths for EUI. The instrument subsystems (detectors, filters, mirrors) have been characterised over a very large wavelength range in order to determine their spectral response not only at the working wavelength but also outside the spectral band of interest. The model of the combined efficiencies of the subsystems was then compared with the response of the instrument derived in the end-to-end test.

The end-to-end calibration of the complete instrument was made in a dedicated vacuum tank at PTB that could be mounted at the beamline of the MLS. Future papers are in preparation that will provide in-depth information on the ground calibration results of the specific performance assessment and calibration results of each telescope (Auchère \& EUI consortium partners, in prep.; Schühle \& EUI consortium partners, in prep.; Gissot \& EUI consortium partners, in prep.).

\section{Concepts of operations}

In contrast to most previous solar space observatories, Solar Orbiter should be thought of as a deep-space encounter mission with observation planning dictated by opportunities brought by the regularly changing orbits. Full operation of the remotesensing instrument package of Solar Orbiter, including EUI, will be restricted to three periods of ten days per 168 days orbit, corresponding to perihelion and to maximal solar latitude north and south. In between these three periods, EUI will take part in a program of much reduced, synoptic remote sensing observations (Auchère et al. 2020).

Each day in the mission will therefore provide a different, if not unique, science observation opportunity as a combination of 
the solar distance (hence spatial resolution of the EUI images), solar latitude (hence opportunities to observe the solar poles), solar activity (as the solar cycle progresses), and relative position with respect to Earth and other observatories (e.g. for joint campaigns during quadratures). As every day in the mission provides unique opportunities, science planning from mission timescales down to individual observations is essential (see Sect. 9.3) and must be coordinated across all Solar Orbiter instruments and collaborating observatories.

As a deep space mission in the inner heliosphere, particular constraints apply to Solar Orbiter with respect to telemetry. The telemetry can be interrupted during extended periods (Solar Orbiter-Sun-Earth coalignments) and is strongly limited both in the upward and downward direction. For EUI, this implies strict requirements on the instrument autonomy (Sect. 9.1), onboard calibration functionalities (Sect. 9.4), and on the ability of the instrument to prioritise the most important subset of the produced data (Sect. 9.5).

\subsection{Autonomy}

Because of the limited availability of ground stations, the light travel time to the spacecraft, and frequent conjunctions of the spacecraft and the Sun with Earth, there is nominally no realtime commanding or continuous communication contact with Solar Orbiter and hence with EUI. Therefore, the EUI operations software is designed to fix or work around problems itself and keep the instrument observing. The software retries on communication errors and mechanism moves, skips incorrect exposures, and reboots and resets the cameras if they malfunction. Reliability is good on the ground but the radiation environment of deep space will inevitably cause data errors and slow degradation.

Observing is mainly autonomous as only 150 telecommands are allowed per day. All nominal observation programs (see Sect. 9.2) and onboard calibration programs (see Sect. 9.4) are stored in the CEB memory as configuration tables (also called Science Tables) containing all required exposure sequences and parameter sets. As these Science Tables also include loops and waits, they fully characterise the observation programs. As a result, once ready in "Sci" mode, the software requires only a single telecommand to start an observation program on each EUI telescope. Throughout the mission, these tables can be modified, either adapting parameters for a specific observation program, or adding extra observing programs. The Science Tables thus provide both autonomy and flexibility.

\subsection{The EUI observation programs}

As explained above, the EUI observation programs are defined in onboard Science Tables that can be executed with a single telecommand and that can be modified at any time through dedicated telecommands. At the time of instrument delivery, the following sample observation programs were loaded in the Science Tables of the CEB:

- The Synoptic program takes FSI $17.4 \mathrm{~nm}$ and $30.4 \mathrm{~nm}$ images at 15 min cadence, binned $2 \times 2$ and with a high compression (0.32 bits per pixel). This program is intended to be running continuously during all Remote Sensing Windows.

- The Global Eruptive Event program takes FSI $17.4 \mathrm{~nm}$ or $30.4 \mathrm{~nm}$ images at $10 \mathrm{~s}$ cadence without binning and with a low compression (1.20 bits per pixel), and is well-suited to studying dimmings and EIT waves for example.

- The Faint High Corona program integrates 60 one-minute exposures in FSI $17.4 \mathrm{~nm}$ or $30.4 \mathrm{~nm}$ into a long one-hour expo- sure, with the FSI occulter in place. It is intended to study the faint high corona. The individual one-minute exposures can optionally be scrubbed for particle hits before integrating them in the long one-hour image. As in any other program, the Faint High Corona program is fully configurable and will need to be carefully optimised during commissioning to balance $\mathrm{S} / \mathrm{N}$ with time resolution for specific observational goals.

- The Coronal Hole program takes HRI $\mathrm{EUV}_{\mathrm{V}}$ and/or $\mathrm{HRI}_{\mathrm{Lya}}$ images at $30 \mathrm{~s}$ cadence without binning and with a very low compression (2.40 bits per pixel). These parameters have been selected to get enough $\mathrm{S} / \mathrm{N}$ in the dark coronal hole regions.

- The Discovery program takes full-FOV HRI $\mathrm{EUV}_{\mathrm{E}}$ images at $1 \mathrm{~s}$ cadence and/or partial-FOV $\mathrm{HRI}_{\text {Lya }}$ images at subsecond cadence without binning and with a medium compression ( 0.80 bits per pixel). This program is intended for discovery science at very high spatial and temporal resolution.

- The Find Event program takes FSI $17.4 \mathrm{~nm}$ or $30.4 \mathrm{~nm}$ images at 1 min cadence, binned $4 \times 4$ and with a very high compression ( 0.12 bits per pixel), and employs the thumbnails to detect major solar eruptions (see Sect. 9.5). This program can be used in combination with for example, the Discovery program, resulting in very-high-cadence HRI observations of a solar eruption if a flare is detected.

- The Beacon program takes FSI $17.4 \mathrm{~nm}$ and $30.4 \mathrm{~nm}$ images at 15 min cadence, binned $4 \times 4$ and with a very high compression ( 0.12 bits per pixel). Beacon images are sent to the ground in the next ground station pass as low-latency telemetry (see Sect. 9.7), and serve as context for the ground segment for optimal planning of operations.

We note that the mentioned parameters are only initial guesses and will be optimised during commissioning when real coronal EUI images become available.

\subsection{Planning concept}

The EUI science planning is strongly rooted in the overall Solar Orbiter planning process (see Zouganelis et al. 2020; Auchère et al. 2020 for a full description). Here we focus on some EUI specific aspects.

In the long term, the coordination of multi-instrument campaigns is defined by Solar Orbiter Observing Plans (SOOPs), the scheduling of which is frozen at least 6 months before execution. A SOOP is a collection of instrument operations that belong together as they serve a common science goal. In the months before the execution, the precise contribution of EUI to a SOOP is defined through Instrument Operation Requests (IORs).

The IORs will define when the above research programs will be scheduled and will also clarify the impact the EUI operations will have in terms of power usage, telemetry needs, and, possibly, electromagnetic noise (EMC). There will be one IOR per day, sent to the spacecraft in groups of seven once per week. Following an analysis of these aspects by the Science Operations Centre (SOC: Sanchez et al., in prep.), the EUI activities as defined in an IOR are mostly frozen four weeks before the execution. The exception to this are minor changes to the instrument operations for which no impact is expected beyond EUI (the socalled resource-neutral parameters) such as altering image priorities, detector settings, or algorithm configurations. Another important exception is the spacecraft pointing. Given the small FOV of the EUI HRIs, it is of course not feasible to define four weeks in advance where on the Sun an appropriate target will be located. Therefore, in some SOOPs it will be possible to make updates to the desired spacecraft pointing. As spacecraft pointing affects all instruments at once, such updates are defined by 
the SOOP coordinator, typically a scientist co-located at the SOC premises and in close contact with all the contributing instrument teams. As any update sent to the spacecraft needs to be first double checked and then channelled through daily contacts, the last updates to instrument operations or spacecraft pointing need to be defined a few days in advance of the execution.

External users of EUI data are most welcome to contact the EUI consortium and suggest specific operations for the EUI instrument but need to be aware of the long-lead time nature of Solar Orbiter EUI science planning sketched out above, which is significantly different from that of other solar observatories.

In a sense, the planning process is not finished after the observations have been acquired and written to the EUI internal memory (OHQ or SOB). Each image will be labelled with a priority number and images with the least priority (highest priority number) will be overwritten when the internal memory is full. A default priority number will be assigned a priori which can encode for example that certain data (e.g. synoptics) should never be overwritten, while other data should only be brought to the ground if telemetry allows. Onboard algorithms can alter the default priority of images depending on their quality (e.g. deprioritise blurred images) or depending on their content (e.g. prioritise images corresponding to an eruption). Finally, the priority can also be changed from the ground through telecommand to bring down a maximum amount of data corresponding to an unexpected, noteworthy event for example.

\subsection{Onboard calibration}

During most of the orbits (except for the 30 days of Remote Sensing Windows (RSWs) per orbit and the low telemetry outof-RSW synoptic FSI program), the EUI telescopes will be unused and will undergo deep thermal cycling. Therefore, a fresh assessment of the performance of the telescopes must be made at the beginning of each orbit, and preferentially also at the end of each ten-day RSW. This assessment has two goals: onboard calibration and detector degradation monitoring. For both goals, the assessment will consist of taking many $(N>30)$ dark and LED images at a number of exposure times and optionally in fast sequence to determine noise statistics. Additional calibration observations might take advantage of spacecraft rolls or dynamic blurring. As it is not possible to bring everything to the ground for processing, the calibration exposures will be stored in the One Hour Queue (OHQ) and processed by the software onboard to produce small calibration summaries for the ground and large calibration maps to be stored in the non-volatile flash memory.

Onboard, the calibration information will be turned into correction tables containing, for each pixel, the offset-value and the gain factor, which are applied to the image values before they are compressed. In principle, the correction tables could fully correct for offset and flat field. The objective of the onboard correction tables is however limited to correcting strong pixel-to-pixel variability as this critically affects compression. Any smoothly varying calibration component (e.g. vignetting) can always be corrected post-decompression on the ground.

Once the quality of a calibration has been verified on the ground, it can be used and will be copied by the software to the Compression SRAM ready for use by the Compression Board FPGA. The full cycle of an onboard calibration update will therefore consist of the following sequence of operations:

1. Just before or at the start of the first RSW of an orbit, EUI runs a calibration update, thereby collecting hundreds of images. Onboard EUI software turns this large number of images into summary statistics and tables.
2. A representative set of these are brought to the ground for validation.

3. The EUI team inspects the downlinked calibration information.

4. If the newly derived tables are appropriate, they will be approved for onboard application by an instrument configuration telecommand that specifies to use the new table instead of a previously existing table.

5. All images taken by EUI from then on are corrected by the new tables before compression.

\subsection{Event detection}

The second and third overarching science questions of Solar Orbiter (see Sect. 1) refer explicitly to solar eruptions. Given frequent and non-stop imaging, FSI will easily observe the development of flares and filament eruptions and associated EIT waves, CMEs, and other phenomena. We anticipate that such FSI observations will be essential in multi-instrument campaigns aiming at connection science.

However, on theoretical grounds, it is obvious that small length scales are at the heart of the energy-release process in eruptions, making high-resolution observation of the early phase of eruptions mandatory. Unfortunately, given the small FOV, it is far more difficult to observe the origin of eruptions with the HRIs. This problem is aggravated by the limited telemetry which implies that the HRIs can only be operated at appropriate cadences during limited time-spans. Besides pointing the spacecraft to the most dynamical active region (see also Sect. 9.3), the chance of "catching" an eruption in the HRI FOV will be optimised through reprioritising the download of buffered images. Two versions of this strategy will be employed: (1) one driven through ground control and (2) another driven by onboard event detection. In the first case, the idea is to extend the duty cycle of the HRIs as much as possible, and deprioritise (i.e. make them available for overwriting) by telecommand if on the ground we have assessed from low-latency images (see Sect. 9.7) that no significant activity took place. In the second case, the FSI camera takes an image and produces the corresponding thumbnail every minute, while the HRI cameras are observing in very high cadence (around $1 \mathrm{~s}$ ). The FSI thumbnails are fed into an onboard algorithm that checks for the occurrence of large eruptions. The algorithm is based on SOFAST (Bonte et al. 2013) and essentially triggers when a brightness threshold is exceeded in differences between subsequent thumbnail images. The algorithm has various free parameters that will need to be optimised in flight. The OHQ is filled and cyclically overwritten perhaps multiple times (approximately every hour) with the incoming images. If an event is found and is inside the FOV of the HRIs, the HRI data from some time before and for some time after are automatically preserved (and given a higher priority based on the significance of the event) by copying them from the OHQ to the SOB for longer-term storage. A subsequent campaign may find a more interesting or less interesting event (decided by parameters of the event detection algorithm) and would be tagged with lower or higher priority, respectively. Astronomers on the ground can look at low-latency telemetry to help decide whether or not a sequence of images is worth sending to the ground, and can manually reprioritise the images by telecommand after the detection but before the images are sent down.

Just as other instruments notify EUI of events that they have detected, EUI notifies the other instruments and itself via (onboard) IIC when it is hunting for events and has detected one. This allows coordinated observations between several 
instruments. Parameters of the EUI event detection are included in the IIC message. As EUI has very limited telemetry available, the science data are prioritised with initial priorities from the science tables that can be modified by telecommands from ESA on the ground or autonomously by the onboard software based on image quality or IIC data. In this way, poor-quality data can be discarded and the data around the time of an interesting event can be re-prioritised and made more likely to be sent to the ground when contact is made and telemetry becomes available.

\subsection{The EUI Data Centre}

The EUI Data Centre (EDC) is located at the Royal Observatory of Belgium (ROB), and is responsible for instrument operation planning, monitoring of instrument health, and processing of the instrument telemetry to various data products. The EDC is not an "operational" centre, that is, no commands will be sent directly to the instrument from the EDC. Instead the SOC is responsible for the overall Solar Orbiter scientific mission planning and experiment command request preparation (including EUI), to be submitted in a consolidated manner to the MOC (Mission Operations Centre) in Darmstadt.

The EDC consists of both software developed at ROB and operators who interact with that software for proper planning and monitoring. The software tools developed for the EDC include a Scenario Editor and Simulator in which the operator can create EUI observation plans and simulate their effects, an IOR Executor which translates the scenarios into the lists of telecommands needed by the spacecraft, a Mission Planning Viewer which depicts spacecraft activities, and a Housekeeping Timeline Viewer which can show timelines of almost 2000 housekeeping parameters such as detector temperatures.

\subsection{Data products and data distribution policy}

The EUI data products will be fully compliant with the standard agreed by the Solar Orbiter instruments in the Modelling and Data Analysis Working Group (Solar Orbiter MADAWG 2015; Rouillard et al. 2020). As such, the following levels in EUI science data products will be distinguished:

- Level 0: "Raw" data. This is unpacked and decompressed data, stored in FITS files with limited metadata reflecting the information that was available in the science telemetry packets only. As the metadata is incomplete, the usage of this data product outside the EUI Data Centre is not recommended.

- Level 1: "Engineering" data. This is uncalibrated data, stored in FITS files with completed metadata following the Solar Orbiter standard for L1. The usage of this data product is only recommended for users who want to see the raw, original data in order to study or improve the EUI calibration procedures for example.

- Level 2: "Calibrated" data. This is science-quality data stored in FITS files and metadata following the Solar Orbiter standard for L2. This data product is calibrated and corrected for all instrumental defects known to the EUI consortium at the time of production of the data product. This data product is therefore recommended for all quantitative research applications.

- Level 3: "Visualisation" data. All EUI images will be processed as JPEG2000 images for visualisation through the JHelioviewer tool (Müller et al. 2017). This data product is not suitable for quantitative research applications but is intended as easy access for qualitative and morphological assessments, for all exploratory, quicklook visual needs, and for outreach purposes.
The above science data products will be produced by the EUI Data Centre, typically within a few hours following the arrival of the data on the ground. However, we note that given the deep space nature of Solar Orbiter, significant delays (up to many months) are possible between the observation time and the data arrival on the ground.

The need for a faster data turn-around for Solar Orbiter planning purposes, but also for space weather monitoring, is addressed by the low-latency data stream (Solar Orbiter SOC 2017). The low-latency EUI data is a subset of all EUI data, which is typically strongly compressed and is routed with priority and processed on the ground by a fast, simplified processing pipeline directly at the Science Operations Centre at ESAC. In this way, the EUI low-latency data will become available with the shortest possible time delay, typically within $24 \mathrm{~h}$ of the observation time. We note that the usage of EUI images produced by the low-latency pipeline at ESAC is only recommended for time critical purposes. For non-time critical purposes, it is important to note that on slightly longer production time-scales, all low-latency EUI data will also be processed by the full science calibration pipeline at the EUI Data Centre and be distributed as regular science products.

The EUI consortium is committed to a maximally open data policy meaning that all data products, low-latency data products, the calibration software, and science user documentation will be made available as soon as possible and to as broad an audience as possible. This implies specifically that the EUI consortium will not exercise the right foreseen in the Solar Orbiter Management Plan (Solar Orbiter 2012) to retain the data for the 3 months following the receipt of the original telemetry for the purpose of calibration and verification. Instead, the EUI data products will be distributed as soon as is technically possible with the best known calibration at the time of production.

The full EUI data archive will be reprocessed on a regular basis to apply the continuously improving instrument understanding and calibration optimisation. Each such reprocessing will be distributed as a new release of the data. Typically only Level 2 data will be upgraded in a new release. Level 0 data will never change by definition. Level 1 data will only be upgraded if the metadata content is changing. Level 3 data will only upgrade if the changes imply a visually perceptible change. Previous versions of the data corresponding to old releases will continue to be available for reproducibility of past research.

As the EUI data are immediately released with the best available calibration, some artifacts might remain in the data until they are resolved in a later release. Scientists outside the EUI consortium are therefore encouraged to contact the EUI team for specific advice and support when using EUI data. Co-authorship will be requested depending on the level of involvement of EUI team members.

All EUI science data will be available from the EUI Data Centre and from the Solar Orbiter Archive (SOAR) ${ }^{12}$. The EUI science data will be further propagated through Virtual Observatories (Rouillard et al. 2020) and online visualisation platforms such as JHelioviewer (Müller et al. 2017).

\section{Conclusions}

The EUI instrument as described in this paper was delivered to Airbus UK in May 2017 for integration on the Solar Orbiter platform.

12 http://soardev.esac .esa.int/soar-beta/ 
The EUI instrument is designed as a general-purpose imager of the solar atmosphere, from chromosphere to extended corona and from the highest spatial resolution to the broadest FOV ever achieved. As such, the expected science outcome of EUI is very broad. Together with the other instruments onboard Solar Orbiter, EUI will take part in joint campaigns to fullfil the Solar Orbiter objectives, but will also pursue its own research goals.

Future papers are in preparation that will give in-depth information for each telescope on the ground calibration results and in flight specific performance assessment (Auchère \& EUI consortium partners, in prep.; Schühle \& EUI consortium partners, in prep.; Gissot \& EUI consortium partners, in prep.).

Acknowledgements. EUI was conceived by a multi-national consortium and proposed in 2008 under the scientific lead of Royal Observatory of Belgium (ROB) and the engineering lead of Centre Spatial de Liège (CSL). The proposal was led by Jean-François Hochedez (ROB) and, after acceptance, Pierre Rochus of CSL became the Principal Investigator in 2009. He was assisted by Consortium Project Manager Etienne Renotte and EUI System Engineer Jean-Philippe Halain until instrument delivery, when it was handed over to Aline Hermans. The Common Electronic Box of EUI was contributed by the UCL Mullard Space Science Laboratory (MSSL) under the lead of institute project manager Tom Kennedy, with Jason Tandy, Kyle Silliman and Sampie Smit responsible for the electronics design and Phil Smith responsible for the software design. Louise Harra was the co-principal investigator at MSSL until mid 2019. Alan Smith took over this role until launch, when David Long will be the co-principal investigator. The Full Sun Imager was designed and developed at Institut d'Astrophysique Spatiale (IAS) under the responsibility of Thierry Appourchaux, replaced as co-PI by Frédéric Auchère after 2013, with Frédéric Rouesnel, Jean-Jacques Fourmond and Cydalise Dumesnil as successive institute project managers. The mirrors of FSI and HRI $\mathrm{EUV}_{\mathrm{EV}}$ were manufactured by Institut d'Optique (IO) under the responsibility of institute project manager Raymond Mercier, with Franck Delmotte leading the development of the multi-layer coatings. The HRI Lya was developed by Max Planck Institute for Solar System Research (MPS) under the lead of Udo Schühle as co-principal investigator, with Michael Kahle, Stefan Meining, and Regina Aznar Cuadrado as successive institute project managers, Klaus Heerlein as lead electronic engineer, and Luca Teriaca as scientific contributor. Physikalisch-Meteorologisches Observatorium Davos/World Radiation Center (PMOD/WRC) contributed the structure of the OBS under the lead of Werner Schmutz as co-principal investigator until 2019 and Louise Harra thereafter. Daniel Pfiffner and Manfred Gyo were the successive institute project managers. Erik Pylyser, Ali BenMoussa and Cis Verbeeck were the successive institute project managers at ROB. Andrei Zhukov is the EUI project scientist. ROB contributed expertise during the development of the CMOS sensors (Boris Giordanengo, Samuel Gissot). Susanna Parenti contributed to the science planning concepts. Benjamin Mampaey, Koen Stegen and Emil Verbeeck contributed to the EUI Data Centre Ground software. The EUI Data Centre will support the science planning and instrument operations after launch, when David Berghmans (ROB) will become the Principal Investigator. The above institutes have received project-specific funding in each of their countries from respectively the Belgian Federal Science Policy Office (BELPSO); the Centre National d'Etudes Spatiales (CNES); the UK Space Agency (UKSA); the Deutsche Zentrum für Luft- und Raumfahrt e.V. (DLR); and the Swiss Space Office (SSO).

\section{References}

Antonucci, E., Romoli, M., Andretta, V., et al. 2020, A\&A, 642, A10 (Solar Orbiter SI)

Auchère, F., \& Artzner, G. E. 2004, Sol. Phys., 219, 217

Auchère, F., Song, X., Rouesnel, F., et al. 2005, in Solar Physics and Space Weather Instrumentation, eds. S. Fineschi, \& R. A. Viereck, Proc. SPIE, 5901, 298

Auchère, F., Ravet-Krill, M.-F., Moses, J. D., et al. 2007, Proc. SPIE, 6689, 66890A

Auchère, F., Rizzi, J., Philippon, A., \& Rochus, P. 2011, J. Opt. Soc. Am. A, 28, 40

Auchère, F., Andretta, V., Antonucci, E., et al. 2020, A\&A, 642, A6 (Solar Orbiter SI)

BenMoussa, A., Gissot, S., Schühle, U., et al. 2013, Sol. Phys., 288, 389

Bonte, K., Berghmans, D., De Groof, A., Steed, K., \& Poedts, S. 2013, Sol. Phys., 286, 185

Chintzoglou, G., De Pontieu, B., Martínez-Sykora, J., et al. 2018, ApJ, 857, 73
Cirtain, J. W., Golub, L., Winebarger, A. R., et al. 2013, Nature, 493, 501 De Pontieu, B., Title, A. M., Lemen, J. R., et al. 2014, Sol. Phys., 289, 2733

Defise, J. M., Clette, F., \& Auchère, F. 1999, in EUV, X-Ray, and Gamma-Ray Instrumentation for Astronomy X, eds. O. H. Siegmund, \& K. A. Flanagan, Proc. SPIE, 3765, 341

Delaboudinière, J.-P., Artzner, G. E., Brunaud, J., et al. 1995, Sol. Phys., 162, 291

Delmotte, F., Meltchakov, E., de Rossi, S., et al. 2013, in Solar Physics and Space Weather Instrumentation V, Proc. SPIE, 8862, 88620A

Garcia-Marirrodriga, C., Pacros, A., Strandmoe, S., et al. 2020, A\&A, in press, https://doi .org/10.1051/0004-6361/202038519 (Solar Orbiter SI)

Gautier, J., Delmotte, F., Françoise Ravet, M., et al. 2008, Opt. Commun., 281, 3032

Green, L. M., Török, T., Vršnak, B., Manchester, W., \& Veronig, A. 2018, Space Sci. Rev., 214, 46

Halain, J.-P., Berghmans, D., Seaton, D. B., et al. 2013, Sol. Phys., 286, 67

Halain, J. P., Mazzoli, A., Meining, S., et al. 2015, in Solar Physics and Space Weather Instrumentation VI, Proc. SPIE, 9604, 96040H

Handy, B. N., Acton, L. W., Kankelborg, C. C., et al. 1999, Sol. Phys., 187, 229

Harra, L., Baker, D., Edwards, S. J., et al. 2015, Sol. Phys., 290, 3203

Hecquet, C., Delmotte, F., Ravet-Krill, M.-F., et al. 2009, Appl. Phys. A: Mater. Sci. Process., 95, 401

Horbury, T., O’Brien, H., Carrasco Blazquez, I., et al. 2020, A\&A, 642, A9 (Solar Orbiter SI)

Kobayashi, K., Cirtain, J., Winebarger, A. R., et al. 2014, Sol. Phys., 289, 4393

Korendyke, C. M., Vourlidas, A., Cook, J. W., et al. 2001, Sol. Phys., 200, 63

Kuzin, S. V., Zhitnik, I. A., Shestov, S. V., et al. 2011, Sol. Syst. Res., 45, 162

Lemen, J. R., Title, A. M., Akin, D. J., et al. 2012, Sol. Phys., 275, 17

Masson, S., McCauley, P., Golub, L., Reeves, K. K., \& DeLuca, E. E. 2014, ApJ, 787,145

Meltchakov, E., Hecquet, C., Roulliay, M., et al. 2010, Appl. Phys. A: Mater Sci. Process., 98, 111

Meltchakov, E., Ziani, A., Auchère, F., et al. 2011, SPIE, 8168, 816819

Meltchakov, E., De Rossi, S., Mercier, R., et al. 2013, in Damage to VUV, EUV, and X-ray Optics IV; and EUV and X-ray Optics: Synergy between Laboratory and Space III, Proc. SPIE, 8777, 87771C

Moses, J. D., Antonnucci, E., Auchère, F., et al. 2020, Nat. Astron., submitted

Müller, D., Nicula, B., Felix, S., et al. 2017, A\&A, 606, A10

Müller, D., St. Cyr, O. C., Zouganelis, I., et al. 2020, A\&A, 642, A1 (Solar Orbiter SI)

Nicula, B., Berghmans, D., \& Hochedez, J.-F. 2005, Sol. Phys., 228, 253

O’Hara, J. P., Mierla, M., Podladchikova, O., D’Huys, E., \& West, M. J. 2019, ApJ, 883, 59

Pant, V., Datta, A., \& Banerjee, D. 2015, ApJ, 801, L2

Reva, A. A., Ulyanov, A. S., Shestov, S. V., \& Kuzin, S. V. 2016, ApJ, 816, 90

Robbrecht, E., Verwichte, E., Berghmans, D., et al. 2001, A\&A, 370, 591

Romoli, M., Capobianco, G., Da Deppo, V., et al. 2007, in Second Solar Orbiter Workshop, ESA SP, 641, 79

Rouillard, A. P., Pinto, R. F., Vourlidas, A., et al. 2020, A\&A, 642, A2 (Solar Orbiter SI)

Schühle, U., Halain, J. P., Meining, S., \& Teriaca, L. 2011, in Solar Physics and Space Weather Instrumentation IV, Proc. SPIE, 8148, 81480K

Schühle, U., Teriaca, L., Aznar Cuadrado, R., et al. 2018, Proc. SPIE, 10699, 1069934

Seaton, D. B., Berghmans, D., Nicula, B., et al. 2013a, Sol. Phys., 286, 43

Seaton, D. B., Groof, A. D., Shearer, P., Berghmans, D., \& Nicula, B. 2013b, ApJ, 777, 72

Slemzin, V., Bougaenko, O., Ignatiev, A., et al. 2008, Ann. Geophys., 26, 3007

Solanki, S. K., del Toro Iniesta, J. C., Woch, J., et al. 2020, A\&A, 642, A11 (Solar Orbiter SI)

Solar Orbiter 2012, Solar Orbiter Science Management Plan, Tech. rep., ESA

Solar Orbiter MADAWG 2015, Metadata Definition for Solar Orbiter Science Data, Tech. rep., ESA

Solar Orbiter SOC 2017, Solar Orbiter Interface Control Document for Low Latency Data FITS Files, Tech. rep, ESA

SPICE Consortium (Anderson, M., et al.) 2020, A\&A, 642, A14 (Solar Orbiter $\mathrm{SI}$

Stenflo, J. O. 2013, A\&ARv, 21, 66

Tadikonda, S. K., Freesland, D. C., Minor, R. R., et al. 2019, Sol. Phys., 294, 28

Thompson, B. J., \& Myers, D. C. 2009, ApJS, 183, 225

Vourlidas, A., Sanchez Andrade-Nuño, B., Landi, E., et al. 2010, Sol. Phys., 261 53

Wülser, J. P., Lemen, J. R., \& Nitta, N. 2007, in Solar Physics and Space Weather Instrumentation II, Proc. SPIE, 6689, 668905

Zouganelis, I., De Groof, A., Walsh, A., et al. 2020, A\&A, 642, A3 (Solar Orbiter SI) 